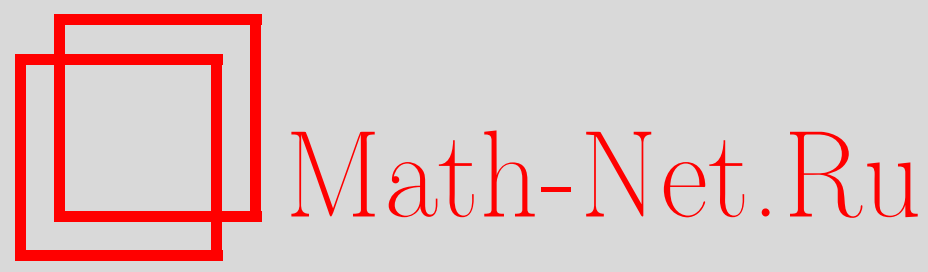

Г. Е. Иванов, Непрерывность оптимальных управлений в дифференциальных играх и некоторые свойства слабо и сильно выпуклых функций, Матем. заметки, 1999, том 66, выпуск 6, 816-839

DOI: https://doi.org/10.4213/mzm1227

Использование Общероссийского математического портала Math-Net.Ru подразумевает, что вы прочитали и согласны с пользовательским соглашением http://www.mathnet.ru/rus/agreement

Параметры загрузки:

IP : 54.205 .225 .156

26 апреля 2023 г., 16:32:18

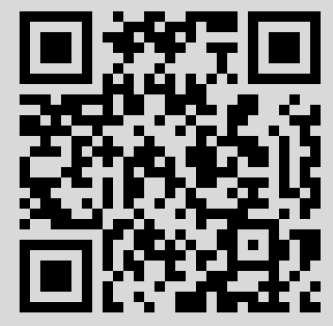




\title{
НЕПРЕРЫВНОСТЬ ОПТИМАЛЬНЫХ УПРАВЛЕНИЙ В ДИФФЕРЕНЦИАЛЬНЫХ ИГРАХ И НЕКОТОРЫЕ СВОЙСТВА СЛАБО И СИЛЬНО ВЫПУКЛЫХ ФУНКЦИЙ
}

\section{Г.Е. Иванов}

\begin{abstract}
В первой части работы доказана эквивалентность липшицевой дифференцируемости функции и совокупности условий слабой выпуклости и слабой вогнутости этой функции, а также приведены достаточные условия непрерывной зависимости седловой точки сильно выпукло-вогнутой функции от параметра. Во второй части доказана гладкость функции цены игры и непрерьвность оптимальных позиционных и программных стратегий игроков в нелинейных дифференциальных играх с нулевой суммой и с сильно выпукло-вогнутьм интегрантом.
\end{abstract}

Библиографояи: 5 названий.

В настоящей работе рассматриваются нелинейные дифференциальные игры с нулевой суммой на фиксированном отрезке времени. Основы теории и некоторые методы решения дифференциальных игр изложены, например, в работах [1]-[4]. В общем случае функция цены дифференциальной игры недифференцируема, что существенно усложняет алгоритмы решения дифференциальных игр. Если же задачу удается решить, то найденные оптимальные стратегии управления являются, как правило, разрьвньпи функциями, что затрудняет практическую реализацию этих стратегий.

Настоящая работа продолжает исследование класса нелинейных дифференщиальных игр, для которых интегрант (подынтегральная функция в интегральном слагаемом функционала качества) является сильно выпукло-вогнутой функцией относительно управлений игроков [5]. Основные результаты настоящей работы состоят в доказательстве гладкости функции цены игры, а также непрерьвности оптимальных позиционных и программных стратегий игроков для рассматриваемого класса дифференциальных игр. В качестве вспомогательных получены результаты, касающиеся некоторых свойств слабо и сильно вьпуклых функций. Доказана эквивалентность липшищевой дифференцируемости функции и совокупности условий слабой вьпуклости и слабой вогнутости этой функции. Приведены достаточные условия непрерьвной зависимости седловой точки сильно выпукло-вогнутой функции от параметра.

Работа выполнена при финансовой поддержке Российского фонда фундаментальных исследований, грант № 98-01-00645. 
1. Обобщенно выпуклые и липшицево дифференцируемые функции. Обозначим через $\mathbb{R}^{s}$ пространство $s$-мерных вектор-столбцов, через

$$
\frac{d g}{d x}\left(x_{0}\right)
$$

строку частных производных (градиент) функции $x \mapsto g(x)$ в точке $x_{0} \in \mathbb{R}^{s}$.

ОПРЕДЕЛЕНИЕ 1.1. Пусть $X \subset \mathbb{R}^{s}$ - выпуклое множество. Будем говорить, что функция $g: \mathbb{R}^{s} \rightarrow \mathbb{R}$ слабо выпукла на множестве $X$ с константой $R$, если функция $x \mapsto g(x)+\frac{1}{2} R\|x\|^{2}$ вьпукла на $X$.

Функция $g$ назьвается сильно выпуклой на множестве $X$ с константой $R>0$, если она слабо выпукла на $X$ с константой $-R$.

Функция $g$ называется слабо (сильно) вогнутой $c$ константой $R$, если $-g$ слабо (сильно) вьпукла с той же константой $R$.

Легко видеть, что функция $g$ слабо вьпуклана $X$ с константой $R$ тогда и только тогда, когда для любых $x_{1}, x_{2} \in X, \lambda \in[0,1]$ вьполнено неравенство

$$
\lambda g\left(x_{1}\right)+(1-\lambda) g\left(x_{2}\right)-g\left(\lambda x_{1}+(1-\lambda) x_{2}\right) \geqslant-\frac{\lambda(1-\lambda)}{2} R\left\|x_{2}-x_{1}\right\|^{2} .
$$

ОПРЕДЕЛЕНИЕ 1.2. Функция назьвается липиицево дифференцируемой с констанmой $L$ на открытом множестве $X$, если она дифференцируема на $X$ и ее градиент удовлетворяет условию Липшица с константой $L$ на множестве $X$.

Теорема 1.1. Пусть $X \subset \mathbb{R}^{n}$ - открытое выпуклое множество. Для того чтобь функиия $g: \mathbb{R}^{n} \rightarrow \mathbb{R}$ была липшицево дифферениируема на множестве $X c$ константой L, необходимо и достаточно, чтобы функиия $g$ была слабо выпукла и слабо вогнута с константой $L$ на $X$.

ДокАЗАТЕЛЬСтво. Необходимость. Пусть $g$ липшицево дифференцируема с константой $L$. Возьмем произвольные $x_{1}, x_{2} \in X, \lambda \in[0,1]$ и обозначим $\bar{x}=\lambda x_{1}+(1-\lambda) x_{2}$. Тогда для любого $i=1,2$ имеем

$$
\begin{aligned}
\left|g\left(x_{i}\right)-g(\bar{x})-\frac{d g}{d x}(\bar{x})\left(x_{i}-\bar{x}\right)\right| & =\left|\int_{0}^{1}\left(\frac{d g}{d x}\left(\mu x_{i}+(1-\mu) \bar{x}\right)-\frac{d g}{d x}(\bar{x})\right)\left(x_{i}-\bar{x}\right) d \mu\right| \\
& \leqslant \frac{1}{2} L\left\|x_{i}-\bar{x}\right\|^{2} .
\end{aligned}
$$

Складьвая эти неравенства при $i=1,2$ с весами $\lambda$ и $1-\lambda$, получим, что функции $g$ и - $g$ удовлетворяют условию (1.1) при $R=L$, т.е. $g$ слабо вьпукла и слабо вогнута с константой $L$.

Достаточность. Для любых $x \in X, \ell \in \mathbb{R}^{n}$ определим производную функции $g$ в точке $x$ по направлению $\ell$ :

$$
F(x, \ell)=\varlimsup_{\lambda \rightarrow+0} \frac{g(x+\lambda \ell)-g(x)}{\lambda} .
$$


Так как $X$ открыто, то для любого $x \in X$ найдется $\delta_{x}>0$ такое, что $\boldsymbol{B}\left(x, \delta_{x}\right) \subset X$, где $\boldsymbol{B}(x, r)$ - открытьй шар в $\mathbb{R}^{n}$ радиуса $r$ с центром в точке $x$.

Для любых $x \in X, \ell \in \boldsymbol{B}\left(0, \delta_{x}\right)$ и $\lambda \in[0,1]$ из формулы (1.1), примененной к функциям $g$ и $-g$ и векторам $x_{1}=x+\ell, x_{2}=x$, получим

$$
|\lambda(g(x+\ell)-g(x))+g(x)-g(x+\lambda \ell)| \leqslant \frac{1}{2} \lambda(1-\lambda) L\|\ell\|^{2} .
$$

Деля на $\lambda$ и переходя к пределу по $\lambda \rightarrow+0$, имеем

$$
|g(x+\ell)-g(x)-F(x, \ell)| \leqslant \frac{1}{2} L\|\ell\|^{2} .
$$

Покажем линейность функции $\ell \mapsto F(x, \ell)$. Зафиксируем произвольные $\ell_{1}, \ell_{2} \in$ $\boldsymbol{B}\left(0, \delta_{x}\right)$. Тог да из $(1.3)$ следует

$$
\begin{gathered}
\left|g\left(x+\ell_{i}\right)-g(x)-F\left(x, \ell_{i}\right)\right| \leqslant \frac{1}{2} L\left\|\ell_{i}\right\|^{2}, \quad i=1,2, \\
\left|g\left(x+\frac{1}{2}\left(\ell_{1}+\ell_{2}\right)\right)-g(x)-F\left(x, \frac{1}{2}\left(\ell_{1}+\ell_{2}\right)\right)\right| \leqslant \frac{1}{2} L\left\|\ell_{1}+\ell_{2}\right\|^{2} .
\end{gathered}
$$

Из (1.1) при $R=L$ вытекает

$$
\left|g\left(x+\ell_{1}\right)+g\left(x+\ell_{2}\right)-2 g\left(x+\frac{1}{2}\left(\ell_{1}+\ell_{2}\right)\right)\right| \leqslant \frac{1}{4} L\left\|\ell_{1}-\ell_{2}\right\|^{2},
$$

откуда

$$
\left|2 F\left(x, \frac{1}{2}\left(\ell_{1}+\ell_{2}\right)\right)-F\left(x, \ell_{1}\right)-F\left(x, \ell_{2}\right)\right| \leqslant \frac{L}{2}\left(\left\|\ell_{1}\right\|^{2}+\left\|\ell_{2}\right\|^{2}+2\left\|\ell_{1}+\ell_{2}\right\|^{2}+\frac{1}{2}\left\|\ell_{1}-\ell_{2}\right\|^{2}\right) .
$$

Поэтому для любых $\ell_{1}, \ell_{2} \in \mathbb{R}^{n} \backslash\{0\}$ и $t, 0 \leqslant t \leqslant \delta_{x} \min \left\{\left\|\ell_{1}\right\|^{-1},\left\|\ell_{2}\right\|^{-1}\right\}$, справедливо неравенство

$$
\left|2 F\left(x, \frac{t}{2}\left(\ell_{1}+\ell_{2}\right)\right)-F\left(x, t \ell_{1}\right)-F\left(x, t \ell_{2}\right)\right| \leqslant \frac{L t^{2}}{2}\left(\left\|\ell_{1}\right\|^{2}+\left\|\ell_{2}\right\|^{2}+2\left\|\ell_{1}+\ell_{2}\right\|^{2}+\frac{1}{2}\left\|\ell_{1}-\ell_{2}\right\|^{2}\right) .
$$

Следовательно, в силу положительной однородности функции $F(x, \cdot)$ получаем

$$
\left|F\left(x, \ell_{1}+\ell_{2}\right)-F\left(x, \ell_{1}\right)-F\left(x, \ell_{2}\right)\right| \leqslant \frac{L t}{2}\left(\left\|\ell_{1}\right\|^{2}+\left\|\ell_{2}\right\|^{2}+2\left\|\ell_{1}+\ell_{2}\right\|^{2}+\frac{1}{2}\left\|\ell_{1}-\ell_{2}\right\|^{2}\right),
$$

т.е. $F\left(x, \ell_{1}+\ell_{2}\right)=F\left(x, \ell_{1}\right)+F\left(x, \ell_{2}\right)$, отсюда и из положительной однородности функции $F(x, \cdot)$ следует линейность этой функции. Поэтому в силу (1.3) функция $g$ дифференцируема по Фреше в точке $x$, значит $g$ дифференцируема на множестве $X$. 
Зафиксируем произвольные $x_{1}, x_{2} \in X, \ell \in \mathbb{R}^{n},\|\ell\|=1$. Для любого натурального $N$ определим

$$
\ell_{N}=\frac{1}{2 N}\left\|x_{2}-x_{1}\right\| \ell
$$

В силу дифференцируемости функции $g$ в точках $x_{1}, x_{2}$ вьполнено

$$
\left(\frac{d g}{d x}\left(x_{1}\right)-\frac{d g}{d x}\left(x_{2}\right)\right) \ell=\lim _{N \rightarrow \infty} \frac{N}{\left\|x_{2}-x_{1}\right\|}\left(g\left(x_{1}+\ell_{N}\right)-g\left(x_{1}-\ell_{N}\right)-g\left(x_{2}+\ell_{N}\right)+g\left(x_{2}-\ell_{N}\right)\right) .
$$

Определим

$$
z_{k}=x_{1}+\frac{k}{N}\left(x_{2}-x_{1}\right), \quad z_{k}^{+}=z_{k}+\ell_{N}, \quad z_{k}^{-}=z_{k}-\ell_{N}, \quad k=0, \ldots, N
$$

Заметим, что

$$
\left|g\left(x_{1}+\ell_{N}\right)-g\left(x_{1}-\ell_{N}\right)-g\left(x_{2}+\ell_{N}\right)+g\left(x_{2}-\ell_{N}\right)\right| \leqslant \sum_{k=0}^{N-1}\left|g\left(z_{k}^{+}\right)-g\left(z_{k}^{-}\right)-g\left(z_{k+1}^{+}\right)+g\left(z_{k+1}^{-}\right)\right| .
$$

Так как функция $g$ слабо вьпукла и слабо вогнута с константой $L$, то

$$
\begin{aligned}
& \left|g\left(z_{k}^{+}\right)+g\left(z_{k+1}^{-}\right)-2 g\left(\frac{1}{2}\left(z_{k}+z_{k+1}\right)\right)\right| \leqslant \frac{1}{4} L\left\|z_{k+1}^{-}-z_{k}^{+}\right\|^{2}, \\
& \left|g\left(z_{k}^{-}\right)+g\left(z_{k+1}^{+}\right)-2 g\left(\frac{1}{2}\left(z_{k}+z_{k+1}\right)\right)\right| \leqslant \frac{1}{4} L\left\|z_{k+1}^{+}-z_{k}^{-}\right\|^{2} .
\end{aligned}
$$

Поэтому

$$
\begin{aligned}
\left|g\left(z_{k}^{+}\right)-g\left(z_{k}^{-}\right)-g\left(z_{k+1}^{+}\right)+g\left(z_{k+1}^{-}\right)\right| & \leqslant \frac{1}{4} L\left(\left\|z_{k+1}^{-}-z_{k}^{+}\right\|^{2}+\left\|z_{k+1}^{+}-z_{k}^{-}\right\|^{2}\right) \\
& =\frac{1}{4} L\left(\left\|\frac{x_{2}-x_{1}}{N}-2 \ell_{N}\right\|^{2}+\left\|\frac{x_{2}-x_{1}}{N}+2 \ell_{N}\right\|^{2}\right) \\
& =\frac{1}{2} L\left(\left\|\frac{x_{2}-x_{1}}{N}\right\|^{2}+4\left\|\ell_{N}\right\|^{2}\right)=\frac{L}{N^{2}}\left\|x_{2}-x_{1}\right\|^{2},
\end{aligned}
$$

откуда с учетом (1.4), (1.5) получаем

$$
\left\|\left(\frac{d g}{d x}\left(x_{2}\right)-\frac{d g}{d x}\left(x_{1}\right)\right) \ell\right\| \leqslant L\left\|x_{2}-x_{1}\right\|
$$

что в силу произвольности единичного вектора $\ell$ дает липшицевость градиента функции $g$ на множестве $X$ с константой $L$. 
2. Непрерывная зависимость седловой точки сильно выпукло-вогнутой функции от параметра. Через $\mathbb{R}^{m \times n}$ обозначим пространство вещественных матриц, имеющих $m$ строк и $n$ столбцов. Если задана дифференцируемая функция $f: \mathbb{R}^{n} \times$ $\mathbb{R}^{m} \rightarrow \mathbb{R}^{k}$ и точки $x_{0} \in \mathbb{R}^{n}, y_{0} \in \mathbb{R}^{m}$, то через

$$
\frac{\partial f}{\partial x}\left(x_{0}, y_{0}\right) \in \mathbb{R}^{k \times n}
$$

будем обозначать матрицу Якоби функции $x \mapsto f\left(x, y_{0}\right)$ в точке $x_{0}$. Если функция $x \mapsto f\left(x, y_{0}\right) \in \mathbb{R}$ выпукла, то через $\partial_{x} f\left(x_{0}, y_{0}\right)$ будем обозначать субдифференциал этой функции в точке $x_{0}$.

Пусть $X, Y, Z$ - непустые множества в нормированных пространствах, а $L$ - вешественное число. Через $\operatorname{Lip}(X, Y ; L)$ обозначим класс функций $f: X \rightarrow Y$, удовлетворяющих условию Липшица на множестве $X$ с константой $L$. Через $\operatorname{BiLip}(X, Y, Z ; L)$ обозначим класс функций $f: X \times Y \rightarrow Z$ таких, что для любых $x_{1}, x_{2} \in X$ и $y_{1}, y_{2} \in Y$ выполняется неравенство

$$
\left\|f\left(x_{1}, y_{1}\right)+f\left(x_{2}, y_{2}\right)-f\left(x_{1}, y_{2}\right)-f\left(x_{2}, y_{1}\right)\right\| \leqslant L\left\|x_{1}-x_{2}\right\| \cdot\left\|y_{1}-y_{2}\right\| .
$$

Заметим, что если $X$ - вьпуклое множество и задана функция $f: X \times Y \rightarrow Z$ такая, что для любого $y \in Y$ функция $f(\cdot, y)$ дифференцируема на множестве $X$ и для любого $x \in X$

$$
\frac{\partial f}{\partial x}(x, \cdot) \in \operatorname{Lip}(Y, Z ; L),
$$

то $f(\cdot, \cdot) \in \operatorname{BiLip}(X, Y, Z ; L)$.

Если $X$ - непустое выпуклое множество в $\mathbb{R}^{s}, R \in \mathbb{R}$, то через $\mathrm{WCo}(X ; R)$ обозначим класс функций $f: X \rightarrow \mathbb{R}$, полунепрерьвных снизу и слабо выпуклых на множестве $X$ с константой $R$. Согласно определению $1.1-\mathrm{WCo}(X ; R)$ - класс функций, полунепрерывных снизу и слабо вогнутых на множестве $X$ с константой $R$. Через $\mathrm{SCo}(X ; R)$ обозначим класс функций $f: X \rightarrow \mathbb{R}$, сильно вьпуклых на множестве $X$ с константой $R>0$ и таких, что функция

$$
x \mapsto \begin{cases}f(x), & x \in X, \\ +\infty, & x \notin X,\end{cases}
$$

полунепрерьвна снизу на $\mathbb{R}^{s}$. Заметим, что в общем случае $\mathrm{WCo}(X ;-R) \neq \mathrm{SCo}(X ; R)$. Например, определив $f(x)=x^{2}$ при $x \in X=(0,1)$, получим, что $f(\cdot) \in \operatorname{WCo}((0,1) ;-1)$, но $f(\cdot) \notin \mathrm{SCo}((0,1) ; 1)$.

Теорема 2.1. Пусть заданы множество $X \subset \mathbb{R}^{m}$, выпуклье множсества $P \subset \mathbb{R}^{p}$, $Q \subset \mathbb{R}^{q}$ и функиия $g: X \times P \times Q \rightarrow \mathbb{R}$. Пусть для любы $x \in X, u \in P, v \in Q$ имеет место

$$
\begin{gathered}
g(x, \cdot, v) \in \operatorname{SCo}\left(P ; R_{u}\right), \quad g(x, u, \cdot) \in-\operatorname{SCo}\left(Q ; R_{v}\right), \\
g(\cdot, \cdot, v) \in \operatorname{BiLip}\left(X, P, \mathbb{R} ; L_{x u}\right), \quad g(\cdot, u, \cdot) \in \operatorname{BiLip}\left(X, Q, \mathbb{R} ; L_{x v}\right) .
\end{gathered}
$$


Для любого $x \in X$ через $u^{s}(x), v^{s}(x)$ обозначим седловую точку функции $(u, v) \mapsto$ $g(x, u, v)$, т.е. $u^{s}(x) \in P, v^{s}(x) \in Q$ и для любых $u \in P, v \in Q$ выполнены неравенства

$$
g\left(x, u^{s}(x), v\right) \leqslant g\left(x, u^{s}(x), v^{s}(x)\right) \leqslant g\left(x, u, v^{s}(x)\right) .
$$

Для любих чисел $a_{1}, a_{2} \geqslant 0 u b_{1}, b_{2}>0$ определим

$$
\varrho\left(a_{1}, a_{2}, b_{1}, b_{2}\right)=\frac{1}{2}\left(\frac{a_{1}}{b_{1}}+\sqrt{\frac{a_{1}^{2}}{b_{1}^{2}}+\frac{a_{2}^{2}}{b_{1} b_{2}}}\right)
$$

и числа $L_{x}^{u}=\varrho\left(L_{x u}, L_{x v}, R_{u}, R_{v}\right), L_{x}^{v}=\varrho\left(L_{x v}, L_{x u}, R_{v}, R_{u}\right)$. Тогда $u^{s} \in \operatorname{Lip}\left(X, P ; L_{x}^{u}\right)$ $u v^{s} \in \operatorname{Lip}\left(X, Q ; L_{x}^{v}\right)$.

ДокАЗАТЕЛЬСтво. Пусть $x_{1}, x_{2} \in X$. Обозначим $u_{i}=u^{s}\left(x_{i}\right), v_{i}=v^{s}\left(x_{i}\right), i=1,2$, $\Delta x=x_{2}-x_{1}, \Delta u=u_{2}-u_{1}, \Delta v=v_{2}-v_{1}$.

Так как $0 \in \partial_{u} g\left(x_{1}, u_{1}, v_{1}\right), 0 \in \partial_{u} g\left(x_{2}, u_{2}, v_{2}\right)$, в силу условий $g\left(x, \cdot, v_{i}\right) \in \operatorname{SCo}\left(P ; R_{u}\right)$, $i=1,2$, имеем

$$
g\left(x_{1}, u_{2}, v_{1}\right)-g\left(x_{1}, u_{1}, v_{1}\right) \geqslant \frac{1}{2} R_{u}\|\Delta u\|^{2}, \quad g\left(x_{2}, u_{1}, v_{2}\right)-g\left(x_{2}, u_{2}, v_{2}\right) \geqslant \frac{1}{2} R_{u}\|\Delta u\|^{2} .
$$

Складьвая два последних неравенства, получаем

$$
g\left(x_{1}, u_{2}, v_{1}\right)+g\left(x_{2}, u_{1}, v_{2}\right)-g\left(x_{1}, u_{1}, v_{1}\right)-g\left(x_{2}, u_{2}, v_{2}\right) \geqslant R_{u}\|\Delta u\|^{2} .
$$

Аналогично, из условий $g\left(x, u_{i}, \cdot\right) \in-\mathrm{SCo}\left(Q ; R_{v}\right), i=1,2$, имеем

$$
g\left(x_{2}, u_{2}, v_{1}\right)+g\left(x_{1}, u_{1}, v_{2}\right)-g\left(x_{1}, u_{1}, v_{1}\right)-g\left(x_{2}, u_{2}, v_{2}\right) \leqslant-R_{v}\|\Delta v\|^{2} .
$$

Следовательно,

$$
g\left(x_{1}, u_{2}, v_{1}\right)+g\left(x_{2}, u_{1}, v_{2}\right)-g\left(x_{2}, u_{2}, v_{1}\right)-g\left(x_{1}, u_{1}, v_{2}\right) \geqslant R_{u}\|\Delta u\|^{2}+R_{v}\|\Delta v\|^{2} .
$$

Из условий $g\left(\cdot, \cdot, v_{1}\right) \in \operatorname{BiLip}\left(X, P, \mathbb{R} ; L_{x u}\right), g\left(\cdot, u_{1}, \cdot\right) \in \operatorname{BiLip}\left(X, Q, \mathbb{R} ; L_{x v}\right)$ получаем

$$
\begin{aligned}
& g\left(x_{1}, u_{2}, v_{1}\right)+g\left(x_{2}, u_{1}, v_{2}\right)-g\left(x_{2}, u_{2}, v_{1}\right)-g\left(x_{1}, u_{1}, v_{2}\right) \\
& \quad \leqslant\left|g\left(x_{1}, u_{2}, v_{1}\right)+g\left(x_{2}, u_{1}, v_{1}\right)-g\left(x_{2}, u_{2}, v_{1}\right)-g\left(x_{1}, u_{1}, v_{1}\right)\right| \\
& \quad+\left|g\left(x_{1}, u_{1}, v_{1}\right)+g\left(x_{2}, u_{1}, v_{2}\right)-g\left(x_{2}, u_{1}, v_{1}\right)-g\left(x_{1}, u_{1}, v_{2}\right)\right| \\
& \quad \leqslant\left(L_{x u}\|\Delta u\|+L_{x v}\|\Delta v\|\right)\|\Delta x\| .
\end{aligned}
$$

Следовательно,

$$
R_{u}\|\Delta u\|^{2}+R_{v}\|\Delta v\|^{2} \leqslant\left(L_{x u}\|\Delta u\|+L_{x v}\|\Delta v\|\right)\|\Delta x\| .
$$

Поэтому

$$
R_{u}\left(\|\Delta u\|-\frac{L_{x u}}{2 R_{u}}\|\Delta x\|\right)^{2}+R_{v}\left(\|\Delta v\|-\frac{L_{x v}}{2 R_{v}}\|\Delta x\|\right)^{2} \leqslant\left(\frac{L_{x u}^{2}}{4 R_{u}}+\frac{L_{x v}^{2}}{4 R_{v}}\right)\|\Delta x\|^{2} .
$$

Таким образом,

$$
\|\Delta u\| \leqslant L_{x}^{u}\|\Delta x\|, \quad\|\Delta v\| \leqslant L_{x}^{v}\|\Delta x\| .
$$

Условия теоремы 2.1 существенно предполагают, что множества $P, Q$ допустимых векторов $u, v$ не зависят от параметра $x$. Следуюшие две теоремы формулируют достаточные условия непрерьвной зависимости седловой точки от параметра в случае, когда множества допустимых векторов $u, v$ зависят от этого параметра. 
ТЕОРема 2.2. Пусть на множестве $\Omega \subset \mathbb{R}^{m}$ заданы многозначные отображения $P, Q$ с непустыми выпуклысми значениями $P(\omega) \subset \mathbb{R}^{p}, Q(\omega) \subset \mathbb{R}^{q}$. Пусть заданы функиии $\alpha: \Omega \times \mathbb{R}^{p} \rightarrow \mathbb{R} \cup\{+\infty\}, \beta: \Omega \times \mathbb{R}^{q} \rightarrow \mathbb{R} \cup\{+\infty\}$ такие, ито для любого $\omega \in \Omega$

$$
\alpha(\omega, \cdot) \in \mathrm{SCo}\left(P(\omega) ; R_{\alpha}^{u}(\omega)\right), \quad \beta(\omega, \cdot) \in \mathrm{SCo}\left(Q(\omega) ; R_{\beta}^{v}(\omega)\right) .
$$

Определим множсества

$$
P_{0}=\operatorname{conv}\left(\bigcup_{\omega \in \Omega} P(\omega)\right), \quad Q_{0}=\operatorname{conv}\left(\bigcup_{\omega \in \Omega} Q(\omega)\right)
$$

где $\operatorname{conv}(M)$ - выпуклая оболочка множества $M$.

Пусть задана функиия $\mu: \Omega \times P_{0} \times Q_{0} \rightarrow \mathbb{R}$ такая, что для любых $\omega \in \Omega, u \in P_{0}$, $v \in Q_{0}$

$$
\mu(\omega, \cdot, v) \in \mathrm{WCo}\left(P_{0} ; R_{u}^{\mu}(\omega)\right), \quad \mu(\omega, u, \cdot) \in-\mathrm{WCo}\left(Q_{0} ; R_{v}^{\mu}(\omega)\right) .
$$

Пусть для любого $\omega \in \Omega$ функиия $(u, v) \mapsto \mu(\omega, u, v)$ дифференцируема на множестве $P_{0} \times Q_{0}$ и для любых $\omega \in \Omega, u \in P_{0}, v \in Q_{0}$

$$
\begin{array}{ll}
\frac{\partial \mu}{\partial u}(\omega, \cdot, v) \in \operatorname{Lip}\left(P_{0}, \mathbb{R}^{1 \times p} ; L_{u u}^{\mu}(\omega)\right), & \frac{\partial \mu}{\partial u}(\omega, u, \cdot) \in \operatorname{Lip}\left(Q_{0}, \mathbb{R}^{1 \times p} ; L_{u v}^{\mu}(\omega)\right), \\
\frac{\partial \mu}{\partial v}(\omega, \cdot, v) \in \operatorname{Lip}\left(P_{0}, \mathbb{R}^{1 \times q} ; L_{u v}^{\mu}(\omega)\right), & \frac{\partial \mu}{\partial v}(\omega, u, \cdot) \in \operatorname{Lip}\left(Q_{0}, \mathbb{R}^{1 \times q} ; L_{u u}^{\mu}(\omega)\right) .
\end{array}
$$

Пусть для любого $\omega \in \Omega$ выполнены неравенства $R_{u}^{\alpha}(\omega)>R_{u}^{\mu}(\omega), R_{v}^{\beta}(\omega)>R_{v}^{\mu}(\omega)$.

Для любого $\omega \in \Omega$ через $\left(u^{s}(\omega), v^{s}(\omega)\right)$ обозначим седловую точку функиии $(u, v) \mapsto$ $\mu(\omega, u, v)+\alpha(\omega, u)-\beta(\omega, v)$.

Зафиксируем точку $\omega_{0} \in \Omega$ и обозначим

$$
u_{0}=u^{s}\left(\omega_{0}\right), \quad v_{0}=v^{s}\left(\omega_{0}\right), \quad \xi_{0}^{u}=-\frac{\partial \mu}{\partial u}\left(\omega_{0}, u_{0}, v_{0}\right), \quad \xi_{0}^{v}=\frac{\partial \mu}{\partial v}\left(\omega_{0}, u_{0}, v_{0}\right) .
$$

Для любого $\omega \in \Omega$ определим

$$
\begin{gathered}
u_{\min }(\omega)=\underset{u \in P(\omega)}{\arg \min }\left(\alpha(\omega, u)-\xi_{0}^{u} u\right), \quad v_{\min }(\omega)=\underset{v \in Q(\omega)}{\arg \min }\left(\beta(\omega, v)-\xi_{0}^{v} v\right), \\
N_{\omega}^{u m}(\omega)=\left\|u_{\min }(\omega)-u_{\min }\left(\omega_{0}\right)\right\|, \quad N_{\omega}^{v m}(\omega)=\left\|v_{\min }(\omega)-v_{\min }\left(\omega_{0}\right)\right\|, \\
N_{u \omega}^{\mu}(\omega)=\left\|\frac{\partial \mu}{\partial u}\left(\omega, u_{0}, v_{0}\right)-\frac{\partial \mu}{\partial u}\left(\omega_{0}, u_{0}, v_{0}\right)\right\|, \\
N_{v \omega}^{\mu}(\omega)=\left\|\frac{\partial \mu}{\partial v}\left(\omega, u_{0}, v_{0}\right)-\frac{\partial \mu}{\partial v}\left(\omega_{0}, u_{0}, v_{0}\right)\right\|, \\
K_{u}(\omega)=N_{u \omega}^{\mu}(\omega)+L_{u u}^{\mu}(\omega) N_{\omega}^{u m}(\omega)+L_{u v}^{\mu}(\omega) N_{\omega}^{v m}(\omega), \\
K_{v}(\omega)=N_{v \omega}^{\mu}(\omega)+L_{u v}^{\mu}(\omega) N_{\omega}^{u m}(\omega)+L_{v v}^{\mu}(\omega) N_{\omega}^{v m}(\omega), \\
N_{\omega}^{u s}(\omega)=N_{\omega}^{u m}(\omega)+\varrho\left(K_{u}(\omega), K_{v}(\omega), R_{u}^{\alpha}(\omega)-R_{u}^{\mu}(\omega), R_{v}^{\beta}(\omega)-R_{v}^{\mu}(\omega)\right), \\
N_{\omega}^{v s}(\omega)=N_{\omega}^{v m}(\omega)+\varrho\left(K_{v}(\omega), K_{u}(\omega), R_{v}^{\beta}(\omega)-R_{v}^{\mu}(\omega), R_{u}^{\alpha}(\omega)-R_{u}^{\mu}(\omega)\right),
\end{gathered}
$$

где функиия @ определена формулой (2.2).

Тогда для любого $\omega \in \Omega$ справедливы неравенства

$$
\left\|u^{s}(\omega)-u^{s}\left(\omega_{0}\right)\right\| \leqslant N_{\omega}^{u s}(\omega), \quad\left\|v^{s}(\omega)-v^{s}\left(\omega_{0}\right)\right\| \leqslant N_{\omega}^{v s}(\omega) .
$$


ДокАЗАТЕЛьСтво. Зафиксируем произвольный параметр $\omega_{*} \in \Omega$ и обозначим

$$
\begin{aligned}
& u_{*}=u^{s}\left(\omega_{*}\right), \quad v_{*}=v^{s}\left(\omega_{*}\right), \quad \xi_{*}^{u}=-\frac{\partial \mu}{\partial u}\left(\omega_{*}, u_{*}, v_{*}\right), \quad \xi_{*}^{v}=\frac{\partial \mu}{\partial v}\left(\omega_{*}, u_{*}, v_{*}\right), \\
& \bar{u}=u_{\min }\left(\omega_{*}\right), \quad \bar{v}=v_{\min }\left(\omega_{*}\right), \quad \bar{\xi}^{u}=-\frac{\partial \mu}{\partial u}\left(\omega_{*}, \bar{u}, \bar{v}\right), \quad \bar{\xi}^{v}=\frac{\partial \mu}{\partial v}\left(\omega_{*}, \bar{u}, \bar{v}\right) .
\end{aligned}
$$

Поскольку $u_{0}=u_{\min }\left(\omega_{0}\right), v_{0}=v_{\min }\left(\omega_{0}\right)$, имеем

$$
\left\|\bar{u}-u_{0}\right\|=N_{\omega}^{u m}\left(\omega_{*}\right), \quad\left\|\bar{v}-v_{0}\right\|=N_{\omega}^{v m}\left(\omega_{*}\right) .
$$

Заметим, что

$$
\begin{aligned}
\left\|\xi_{0}^{u}-\bar{\xi}^{u}\right\| \leqslant & \left\|\frac{\partial \mu}{\partial u}\left(\omega_{0}, u_{0}, v_{0}\right)-\frac{\partial \mu}{\partial u}\left(\omega_{*}, u_{0}, v_{0}\right)\right\|+\left\|\frac{\partial \mu}{\partial u}\left(\omega_{*}, u_{0}, v_{0}\right)-\frac{\partial \mu}{\partial u}\left(\omega_{*}, \bar{u}, v_{0}\right)\right\| \\
& +\left\|\frac{\partial \mu}{\partial u}\left(\omega_{*}, \bar{u}, v_{0}\right)-\frac{\partial \mu}{\partial u}\left(\omega_{*}, \bar{u}, \bar{v}\right)\right\| \\
\leqslant & N_{u \omega}^{\mu}\left(\omega_{*}\right)+L_{u u}^{\mu}\left(\omega_{*}\right)\left\|u_{0}-\bar{u}\right\|+L_{u v}^{\mu}\left(\omega_{*}\right)\left\|v_{0}-\bar{v}\right\| .
\end{aligned}
$$

Отсюда и из (2.6) получаем

$$
\left\|\xi_{0}^{u}-\bar{\xi}^{u}\right\| \leqslant K_{u}\left(\omega_{*}\right) .
$$

Для любых $x \in[0,1], u \in P\left(\omega_{*}\right), v \in Q\left(\omega_{*}\right)$ определим

$$
g(x, u, v)=\mu\left(\omega_{*}, u, v\right)+x\left(\bar{\xi}^{u}-\xi_{0}^{u}\right) u-x\left(\bar{\xi}^{v}-\xi_{0}^{v}\right) v+\alpha\left(\omega_{*}, u\right)-\beta\left(\omega_{*}, v\right) .
$$

Заметим, что для любых $x \in[0,1], u \in P\left(\omega_{*}\right), v \in Q\left(\omega_{*}\right)$ $g(x, \cdot, v) \in \operatorname{SCo}\left(P\left(\omega_{*}\right) ; R_{u}^{\alpha}\left(\omega_{*}\right)-R_{u}^{\mu}\left(\omega_{*}\right)\right), \quad g(x, u, \cdot) \in-\operatorname{SCo}\left(Q\left(\omega_{*}\right) ; R_{v}^{\beta}\left(\omega_{*}\right)-R_{v}^{\mu}\left(\omega_{*}\right)\right)$. Из неравенств (2.7) следует, что для любого $v \in Q\left(\omega_{*}\right)$

$$
g(\cdot, \cdot, v) \in \operatorname{BiLip}\left([0,1], P\left(\omega_{*}\right), \mathbb{R} ; K_{u}\left(\omega_{*}\right)\right) .
$$

Аналогично, для любого $u \in P\left(\omega_{*}\right)$

$$
g(\cdot, u, \cdot) \in \operatorname{BiLip}\left([0,1], Q\left(\omega_{*}\right), \mathbb{R} ; K_{v}\left(\omega_{*}\right)\right) .
$$

Поскольку $0 \in \partial_{u} g\left(0, u_{*}, v_{*}\right), 0 \in \partial_{v}(-g)\left(0, u_{*}, v_{*}\right)$, то $\left(u_{*}, v_{*}\right)$ - седловая точка функции $(u, v) \mapsto g(0, u, v)$. Поскольку $0 \in \partial_{u} g(1, \bar{u}, \bar{v}), 0 \in \partial_{v}(-g)(1, \bar{u}, \bar{v})$, то $(\bar{u}, \bar{v})$ - седловая точка функции $(u, v) \mapsto g(1, u, v)$.

В силу теоремы 2.1 получаем

$$
\left\|u_{*}-\bar{u}\right\| \leqslant \varrho\left(K_{u}\left(\omega_{*}\right), K_{v}\left(\omega_{*}\right), R_{u}^{\alpha}\left(\omega_{*}\right)-R_{u}^{\mu}\left(\omega_{*}\right), R_{v}^{\beta}\left(\omega_{*}\right)-R_{v}^{\mu}\left(\omega_{*}\right)\right),
$$

отсюда и из (2.6) следует неравенство

$$
\left\|u^{s}\left(\omega_{*}\right)-u^{s}\left(\omega_{0}\right)\right\|=\left\|u_{*}-u_{0}\right\| \leqslant N_{\omega}^{u s}\left(\omega_{*}\right) .
$$

Неравенство

$$
\left\|v^{s}\left(\omega_{*}\right)-v^{s}\left(\omega_{0}\right)\right\| \leqslant N_{\omega}^{v s}\left(\omega_{*}\right)
$$

доказьвается аналогично.

Непосредственно из теоремы 2.2 получаем следующую теорему. 
ТЕОРемА 2.3. Пусть выполнены условия теоремы 2.2. Пусть функиии $L_{u и}^{\mu}(\cdot)$, $L_{u v}^{\mu}(\cdot), L_{v v}^{\mu}(\cdot)$ ограничены на мнохестве $\Omega$ и для любого $\omega \in \Omega$ выполнены неравенства

$$
R_{u}^{\alpha}(\omega)-R_{u}^{\mu}(\omega) \geqslant r_{u}>0, \quad R_{v}^{\beta}(\omega)-R_{v}^{\mu}(\omega) \geqslant r_{v}>0
$$

Тогда

а) если функиии

$$
u_{\min }(\cdot), \quad v_{\min }(\cdot), \quad \frac{\partial \mu}{\partial u}\left(\cdot, u_{0}, v_{0}\right), \quad \frac{\partial \mu}{\partial v}\left(\cdot, u_{0}, v_{0}\right)
$$

непрерывны в точке $\omega_{0}$, то функиии $u^{s}(\cdot), v^{s}(\cdot)$ непрерывны в точке $\omega_{0}$;

б) если функиии (2.9) удовлетворяют условию Липиица в точке $\omega_{0}$, то функиии $u^{s}(\cdot), v^{s}(\cdot)$ удовлетворяют условию Липиииа в точке $\omega_{0}$.

Одним из условий теоремы 2.3 является непрерьвность или условие Липшица для функций $u_{\min }, v_{\min }$, определяемых формулами (2.5). Далее приведены достаточные условия на функцию $(s, u) \mapsto \phi(s, u)$ и многозначное отображение $t \mapsto P(t)$, при которых функция

$$
(t, s) \mapsto u_{\min }(t, s)=\underset{u \in P(t)}{\arg \min } \phi(s, u)
$$

непрерьвна или удовлетворяет условию Липшица.

В лемме 2.1 сформулированы достаточные условия, при которьх функция $s \mapsto$ $u_{\min }(t, s)$ удовлетворяет условию Липшица. В лемме 2.2 получены достаточные условия, при которых функция $t \mapsto u_{\min }(t, s)$ непрерьвна или удовлетворяет условию Гёльдера с показателем $1 / 2$. Затем рассмотрен пример, показывающий, что в условиях леммы 2.2 функция $t \mapsto u_{\min }(t, s)$ может не удовлетворять условию Липшица.

Лемма 2.1. Пусть заданы множсество $S \subset \mathbb{R}^{k}$, выпуклое множество $P \subset \mathbb{R}^{p}$ и функиия $\phi: S \times P \rightarrow \mathbb{R}$, удовлетворяющая условиям $\phi(\cdot, \cdot) \in \operatorname{BiLip}(S, P, \mathbb{R} ; L)$, $\phi(s, \cdot) \in \mathrm{SCo}(P ; R)$ для любого $s \in S$. Тогда для функиии

$$
s \mapsto u_{\min }(s)=\underset{u \in P}{\arg \min } \phi(s, u)
$$

выполнено условие $u_{\min }(\cdot) \in \operatorname{Lip}(S, P ; L / R)$.

ДокАЗАтЕЛЬСтво. Обозначим $x=s, X=S, Q=\{0\}, g(x, u, v)=\phi(x, u)$. Тогда для любых $x \in X, u \in P$ вьполнены условия теоремы 2.1 с константами $R_{u}=R$, $R_{v}=1, L_{x u}=L, L_{x v}=0$. Применение теоремы 2.1 дает требуемое утверждение.

Напомним, что расстоянием Хаусдорфа между двумя компактами $\mathscr{A}, \mathscr{B} \in \mathbb{R}^{n}$ называется число

$$
h(\mathscr{A}, \mathscr{B})=\max \left\{\sup _{a \in \mathscr{A}} \inf _{b \in \mathscr{B}}\|a-b\|, \sup _{b \in \mathscr{B}} \inf _{a \in \mathscr{A}}\|a-b\|\right\} .
$$


Лемма 2.2. Пусть на множестве $\mathscr{T}$ задано многозначное отображение $P$ c выпуклы.ми компактными значениями $P(t) \subset \mathbb{R}^{p}$. Пусть на множестве

$$
P_{0}=\operatorname{conv}\left(\bigcup_{t \in \mathscr{T}} P(t)\right)
$$

задана функиия $\phi: P_{0} \rightarrow \mathbb{R}$, удовлетворяющая условиям $\phi(\cdot) \in \operatorname{SCo}\left(P_{0} ; R^{\phi}\right)$ u $\phi(\cdot) \in \operatorname{Lip}\left(P_{0}, \mathbb{R} ; L^{\phi}\right)$. Обозначим

$$
u_{\min }(t)=\underset{u \in P(t)}{\arg \min } \phi(u), \quad t \in \mathscr{T} .
$$

Тогда для любых $t_{1}, t_{2} \in \mathscr{T}$ выполнено неравенство

$$
\left\|u_{\min }\left(t_{1}\right)-u_{\min }\left(t_{2}\right)\right\| \leqslant \sqrt{2 h\left(P\left(t_{1}\right), P\left(t_{2}\right)\right) \frac{L^{\phi}}{R^{\phi}}}+h\left(P\left(t_{1}\right), P\left(t_{2}\right)\right)
$$

ДокАЗАТЕльСтво. Обозначим $u_{i}=u_{\min }\left(t_{i}\right), i=1,2, h=h\left(P\left(t_{1}\right), P\left(t_{2}\right)\right)$. По определению расстояния по Хаусдорфу существует точка $\bar{u}_{2} \in P\left(t_{1}\right)$ такая, что $\left\|\bar{u}_{2}-u_{2}\right\| \leqslant h$. Следовательно,

$$
\phi\left(\bar{u}_{2}\right)-\phi\left(u_{2}\right) \leqslant h L^{\phi} .
$$

Определим функцию

$$
\phi_{1}(u)= \begin{cases}\phi(u), & u \in P\left(t_{1}\right), \\ +\infty, & u \notin P\left(t_{1}\right) .\end{cases}
$$

Тогда $0 \in \partial \phi_{1}\left(u_{1}\right)$, что вместе с сильной вьпуклостью $\phi_{1}$ дает

$$
\phi_{1}\left(\bar{u}_{2}\right) \geqslant \phi_{1}\left(u_{1}\right)+\frac{1}{2} R^{\phi}\left\|\bar{u}_{2}-u_{1}\right\|^{2} \geqslant \phi_{1}\left(u_{1}\right)+\frac{1}{2} R^{\phi}\left(\left\|u_{1}-u_{2}\right\|-h\right)^{2} .
$$

Следовательно, с учетом (2.11)

$$
\phi\left(u_{1}\right)-\phi\left(u_{2}\right) \leqslant h L^{\phi}-\frac{1}{2} R^{\phi}\left(\left\|u_{1}-u_{2}\right\|-h\right)^{2} .
$$

Аналогично,

$$
\phi\left(u_{2}\right)-\phi\left(u_{1}\right) \leqslant h L^{\phi}-\frac{1}{2} R^{\phi}\left(\left\|u_{1}-u_{2}\right\|-h\right)^{2} .
$$

Складывая два последних неравенства, получаем

$$
R^{\phi}\left(\left\|u_{1}-u_{2}\right\|-h\right)^{2} \leqslant 2 h L^{\phi},
$$

т.е.

$$
\left\|u_{1}-u_{2}\right\| \leqslant \sqrt{2 h \frac{L^{\phi}}{R^{\phi}}}+h .
$$


Из леммы 2.2 следует, что если в условиях этой леммы многозначное отображение $P$ удовлетворяет условию Липшища, то функция $u_{\min }$ удовлетворяет условию Гёльдера с показателем $1 / 2$. Следующий пример показьвает, что этот показатель не может быть улучшен.

Пусть $u=(x, y) \in \mathbb{R}^{2}$,

$\phi(u)=\frac{1}{2}\left(x^{2}+y^{2}\right)+y, \quad P(t)=\{(x, y): 0 \leqslant x \leqslant 1,0 \leqslant y \leqslant 1, t-x \sqrt{t} \leqslant y\}, \quad t \in[0,1]$.

Пусть $0 \leqslant t_{1}<t_{2} \leqslant 1$. Тогда $P\left(t_{2}\right) \subset P\left(t_{1}\right)$, так как если $(x, y) \in P\left(t_{2}\right)$, то $t_{1}-x \sqrt{t_{1}} \leqslant$ $\sqrt{t_{1} / t_{2}}\left(t_{2}-x \sqrt{t_{2}}\right) \leqslant \sqrt{t_{1} / t_{2}} y \leqslant y$. Следовательно, $(x, y) \in P\left(t_{1}\right)$. С другой стороны, если $\left(x, y_{1}\right) \in P\left(t_{1}\right)$, то, обозначая $y_{2}=\min \left\{y_{1}+t_{2}-t_{1}, 1\right\}$, получим $\left(x, y_{2}\right) \in P\left(t_{2}\right)$, $\left\|y_{2}-y_{1}\right\| \leqslant\left\|t_{2}-t_{1}\right\|$. Следовательно, отображение $t \mapsto P(t)$ удовлетворяет условию Липшица с константой 1. Функция $\phi$ сильно вьпукла с константой 1.

Зафиксируем произвольное $t \in[0,1]$. Для определения точки минимума $u_{\min }(t)=$ $\left(x_{\min }(t), y_{\min }(t)\right)$ рассмотрим два случая:

1) $x_{\min }(t) \leqslant \sqrt{t}$

2) $x_{\min }(t) \geqslant \sqrt{t}$.

Так как

$$
\frac{\partial \phi(x, y)}{\partial y}=y+1>0
$$

то в случае 1)

$$
\begin{gathered}
y_{\min }(t)=t-x_{\min }(t) \sqrt{t}, \\
\phi_{\min }^{y}(x) \equiv \min _{y:(x, y) \in P(t)} \phi(x, y)=\frac{1}{2}\left(x^{2}+(t-x \sqrt{t})^{2}\right)+t-x \sqrt{t}, \\
\frac{d \phi_{\min }^{y}(x)}{d x}=x-\sqrt{t}(t-x \sqrt{t})-\sqrt{t}=-(1+t)(\sqrt{t}-x) \leqslant 0,
\end{gathered}
$$

следовательно, $x_{\min }(t)=\sqrt{t}$. В случае 2$)$

$$
y_{\min }(t)=0, \quad \phi_{\min }^{y}(x)=\frac{x^{2}}{2}, \quad \frac{d \phi_{\min }^{y}(x)}{d x}=x>0, \quad x_{\min }(t)=\sqrt{t} .
$$

Итак, $x_{\min }(t)=\sqrt{t}, y_{\min }(t)=0$ и показатель $1 / 2$ в условии Гёльдера для функции $u_{\min }$ не может быть улучшен.

3. Дифференциальная игра с сильно выпукло-вогнутым интегрантом. Основные предположения. Рассмотрим дифференциальную игру

$$
\dot{x}(t)=f(t, x(t), u(t), v(t)), \quad x\left(t_{0}\right)=x_{0}
$$

на отрезке времени $t \in\left[t_{0}, T\right], 0 \leqslant t_{0}<T$, с функционалом качества

$$
\mathscr{J}=\gamma(x(T))+\int_{t_{0}}^{T}(\varphi(t, x(t), u(t), v(t))+\alpha(t, u(t))-\beta(t, v(t))) d t
$$


где $u(t) \in P(t) \subset \mathbb{R}^{p}$ - управление первого игрока, стремящегося минимизировать функционал $\mathscr{J}, v(t) \in Q(t) \subset \mathbb{R}^{q}$ - управление второго игрока, максимизирующего $\mathscr{J}$, $x(t) \in \mathbb{R}^{n}-$ фазовый вектор системы.

Будем предполагать, что функции $\alpha:[0, T] \times \mathbb{R}^{p} \rightarrow \mathbb{R} \cup\{+\infty\}, \beta:[0, T] \times \mathbb{R}^{q} \rightarrow$ $\mathbb{R} \cup\{+\infty\}$ борелевские и для любого $t \in[0, T]$ вьполнены условия

$$
\alpha(t, \cdot) \in \mathrm{SCo}\left(P(t) ; R_{u}^{\alpha}(t)\right), \quad \beta(t, \cdot) \in \mathrm{SCo}\left(Q(t) ; R_{v}^{\beta}(t)\right) .
$$

Пусть для любых $\xi^{u} \in \mathbb{R}^{1 \times p}, \xi^{v} \in \mathbb{R}^{1 \times q}$ функции

$$
\begin{gathered}
t \mapsto \alpha^{*}\left(t, \xi^{u}\right)=-\min _{u \in P(t)}\left(\alpha(t, u)-\xi^{u} u\right), \quad t \mapsto \beta^{*}\left(t, \xi^{v}\right)=-\min _{v \in Q(t)}\left(\beta(t, v)-\xi^{v} v\right), \\
t \mapsto u_{\min }\left(t, \xi^{u}\right)=\underset{u \in P(t)}{\arg \min }\left(\alpha(t, u)-\xi^{u} u\right)=\frac{\partial \alpha^{*}}{\partial \xi^{u}}\left(t, \xi^{u}\right), \\
t \mapsto v_{\min }\left(t, \xi^{v}\right)=\underset{v \in Q(t)}{\arg \min }\left(\beta(t, v)-\xi^{v} v\right)=\frac{\partial \beta^{*}}{\partial \xi^{v}}\left(t, \xi^{v}\right)
\end{gathered}
$$

непрерьвны на $[0, T]$.

ОПРЕДЕЛЕНИЕ 3.1. Классом допустимых программных стратегий игрока $и$ будем назьвать множество $\mathscr{U}$ измеримых функций $u:\left[t_{0}, T\right] \rightarrow \mathbb{R}^{p}$ таких, что для любых $t \in\left[t_{0}, T\right] u(t) \in P(t)$ и

$$
\int_{t_{0}}^{T} \alpha(t, u(t)) d t<+\infty .
$$

Классом допустимых программных стратегий игрока $v$ будем назьвать множество $\mathscr{V}$ измеримых функций $v:\left[t_{0}, T\right] \rightarrow \mathbb{R}^{q}$ таких, что для любых $t \in\left[t_{0}, T\right] v(t) \in Q(t)$ и

$$
\int_{t_{0}}^{T} \beta(t, v(t)) d t<+\infty
$$

Заметим, что из сильной вьпуклости функций $\alpha(t, \cdot), \beta(t, \cdot)$ и непрерьвности функций $u_{\min }(\cdot, 0), v_{\min }(\cdot, 0)$ следует, что допустимые программные стратегии являются квадратично интегрируемыми функциями на $\left[t_{0}, T\right]$.

Пусть на отрезке $[0, T]$ задано многозначное отображение $X$ с непустыми выпукльми значениями $X(t)$, график которого $\Omega \equiv\{(t, x) \mid t \in[0, T], x \in X(t)\}$ является открытым множеством в топологии $[0, T] \times \mathbb{R}^{n}$. Будем предполагать, что для любых $t_{0} \in[0, T)$, $x_{0} \in X\left(t_{0}\right), u \in \mathscr{U}, v \in \mathscr{V}$ сушествует решение задачи Коши (3.1), причем $x(t) \in X(t)$ для всех $t \in\left[t_{0}, T\right]$.

Определим множества

$$
P_{0}=\operatorname{conv}\left(\bigcup_{t \in[0, T]} P(t)\right), \quad Q_{0}=\operatorname{conv}\left(\bigcup_{t \in[0, T]} Q(t)\right)
$$


Пусть функция $f: \Omega \times P_{0} \times Q_{0} \rightarrow \mathbb{R}^{n}$ непрерьвна, для любого $t \in[0, T]$ функция $(x, u, v) \mapsto f(t, x, u, v)$ дифференцируема на множестве $X(t) \times P_{0} \times Q_{0}$ и функции $\partial f / \partial u$, $\partial f / \partial v$ непрерьвны на множестве $\Omega \times P_{0} \times Q_{0}$. Пусть для любых $t \in[0, T], x \in X(t)$, $u \in P_{0}, v \in Q_{0}$ выполнены условия

$$
\begin{gathered}
\frac{\partial f}{\partial x}(t, \cdot, u, v) \in \operatorname{Lip}\left(X(t), \mathbb{R}^{n \times n} ; L_{x x}^{f}(t)\right), \\
\frac{\partial f}{\partial u}(t, \cdot, u, v) \in \operatorname{Lip}\left(X(t), \mathbb{R}^{n \times p} ; L_{x u}^{f}(t)\right), \quad \frac{\partial f}{\partial v}(t, \cdot, u, v) \in \operatorname{Lip}\left(X(t), \mathbb{R}^{n \times q} ; L_{x v}^{f}(t)\right), \\
\frac{\partial f}{\partial u}(t, x, \cdot, v) \in \operatorname{Lip}\left(P_{0}, \mathbb{R}^{n \times p} ; L_{u u}^{f}(t)\right), \quad \frac{\partial f}{\partial v}(t, x, \cdot, v) \in \operatorname{Lip}\left(P_{0}, \mathbb{R}^{n \times q} ; L_{u v}^{f}(t)\right), \\
\frac{\partial f}{\partial u}(t, x, u, \cdot) \in \operatorname{Lip}\left(Q_{0}, \mathbb{R}^{n \times p} ; L_{u v}^{f}(t)\right), \quad \frac{\partial f}{\partial v}(t, x, u, \cdot) \in \operatorname{Lip}\left(Q_{0}, \mathbb{R}^{n \times q} ; L_{v v}^{f}(t)\right), \\
\left\|\frac{\partial f}{\partial x}(t, x, u, v)\right\| \leqslant M_{x}^{f}(t), \quad\left\|\frac{\partial f}{\partial u}(t, x, u, v)\right\| \leqslant M_{u}^{f}(t), \quad\left\|\frac{\partial f}{\partial v}(t, x, u, v)\right\| \leqslant M_{v}^{f}(t), \\
\|f(t, x, u, v)\| \leqslant M^{f}+M_{x}^{f}(t)\|x\|+M_{u}^{f}(t)\|u\|+M_{v}^{f}(t)\|v\| .
\end{gathered}
$$

Предполагаем далее, что функция $\gamma: X(T) \rightarrow \mathbb{R}$ удовлетворяет условиям

$$
\gamma(\cdot) \in \mathrm{WCo}\left(X(T) ; R^{+\gamma}\right), \quad \gamma(\cdot) \in-\mathrm{WCo}\left(X(T) ; R^{-\gamma}\right),
$$

причем $R^{+\gamma} \geqslant 0$ и $R^{-\gamma} \geqslant 0$.

Определим константу $N^{\gamma} \in \mathbb{R} \cup\{+\infty\}$ :

$$
N^{\gamma}=\sup _{x \in X(T)}\left\|\frac{d \gamma(x)}{d x}\right\| .
$$

Пусть функция $\varphi: \Omega \times P_{0} \times Q_{0} \rightarrow \mathbb{R}$ непрерьвна, для любого $t \in[0, T]$ функция $(x, u, v) \mapsto \varphi(t, x, u, v)$ дифференцируема на множестве $X(t) \times P_{0} \times Q_{0}$ и функции $\partial \varphi / \partial u$, $\partial \varphi / \partial v$ непрерывны на множестве $\Omega \times P_{0} \times Q_{0}$. Пусть для любых $t \in[0, T], x \in X(t)$, $u \in P_{0}, v \in Q_{0}$ выполнены условия

$$
\begin{array}{cl}
\varphi(t, x, \cdot, v) \in \mathrm{WCo}\left(P_{0} ; R_{u}^{\varphi}(t)\right), & \varphi(t, x, u, \cdot) \in-\mathrm{WCo}\left(P_{0} ; R_{v}^{\varphi}(t)\right), \\
\varphi(t, \cdot, u, v) \in \mathrm{WCo}\left(X(t) ; R_{x}^{+\varphi}(t)\right), & \varphi(t, \cdot, u, v) \in-\mathrm{WCo}\left(X(t) ; R_{x}^{-\varphi}(t)\right),
\end{array}
$$

причем $R_{x}^{+\varphi}(t) \geqslant 0, R_{x}^{-\varphi}(t) \geqslant 0$,

$$
\begin{array}{cl}
\frac{\partial \varphi}{\partial u}(t, \cdot, u, v) \in \operatorname{Lip}\left(X(t), \mathbb{R}^{1 \times p} ; L_{x u}^{\varphi}(t)\right), & \frac{\partial \varphi}{\partial v}(t, \cdot, u, v) \in \operatorname{Lip}\left(X(t), \mathbb{R}^{1 \times q} ; L_{x v}^{\varphi}(t)\right), \\
\frac{\partial \varphi}{\partial u}(t, x, \cdot, v) \in \operatorname{Lip}\left(P_{0}, \mathbb{R}^{1 \times p} ; L_{u u}^{\varphi}(t)\right), & \frac{\partial \varphi}{\partial v}(t, x, \cdot, v) \in \operatorname{Lip}\left(P_{0}, \mathbb{R}^{1 \times q} ; L_{u v}^{\varphi}(t)\right), \\
\frac{\partial \varphi}{\partial u}(t, x, u, \cdot) \in \operatorname{Lip}\left(Q_{0}, \mathbb{R}^{1 \times p} ; L_{u v}^{\varphi}(t)\right), & \frac{\partial \varphi}{\partial v}(t, x, u, \cdot) \in \operatorname{Lip}\left(Q_{0}, \mathbb{R}^{1 \times q} ; L_{v v}^{\varphi}(t)\right), \\
\left\|\frac{\partial \varphi(t, x, u, v)}{\partial x}\right\| \leqslant M_{x}^{\varphi}+L_{x x}^{\varphi}(t)\|x\|+L_{x u}^{\varphi}(t)\|u\|+L_{x v}^{\varphi}(t)\|v\| .
\end{array}
$$


Определим функцию $N_{x}^{\varphi}:[0, T] \rightarrow \mathbb{R} \cup\{+\infty\}:$

$$
N_{x}^{\varphi}(t)=\sup _{\substack{x \in X(t) \\ u \in P_{0}, v \in Q_{0}}}\left\|\frac{\partial \varphi(t, x, u, v)}{\partial x}\right\|
$$

Будем предполагать, что указанные вьше функции

$$
\begin{gathered}
R_{u}^{\alpha}, R_{v}^{\beta}, L_{x x}^{f}, L_{x u}^{f}, L_{x v}^{f}, L_{u u}^{f}, L_{u v}^{f}, L_{v v}^{f}, M_{x}^{f}, M_{u}^{f}, M_{v}^{f} \\
R_{u}^{\varphi}, R_{v}^{\varphi}, R_{x}^{+\varphi}, R_{x}^{-\varphi}, L_{x u}^{\varphi}, L_{x v}^{\varphi}, L_{u u}^{\varphi}, L_{u v}^{\varphi}, L_{v v}^{\varphi}
\end{gathered}
$$

измеримы и ограничены на отрезке $[0, T]$.

Пусть для любого $t \in[0, T]$ выполнены неравенства

$$
R_{u}^{\alpha}(t)-R_{u}^{\varphi}(t) \geqslant R_{u}^{\min }>0, \quad R_{v}^{\beta}(t)-R_{v}^{\varphi}(t) \geqslant R_{v}^{\min }>0
$$

обеспечивающие сильную выпукло-вогнутость интегранта $(u, v) \mapsto \zeta(t, x, u, v)=$ $\varphi(t, x, u, v)+\alpha(t, u)-\beta(t, v)$ функционала качества $(3.2)$.

Определим

$$
\begin{gathered}
e(t)=\exp \left(\int_{t}^{T} M_{x}^{f}(\tau) d \tau\right), \quad t \in[0, T], \\
K_{u u}^{f}=\sup _{t \in[0, T]} \frac{L_{u u}^{f}(t) e(t)}{R_{u}^{\alpha}(t)-R_{u}^{\varphi}(t)}, \quad K_{v v}^{f}=\sup _{t \in[0, T]} \frac{L_{v v}^{f}(t) e(t)}{R_{v}^{\beta}(t)-R_{v}^{\varphi}(t)}, \\
K_{u}^{f}=\int_{0}^{T} \frac{\left(M_{u}^{f}(t) e(t)\right)^{2}}{R_{u}^{\alpha}(t)-R_{u}^{\varphi}(t)} d t, \quad K_{v}^{f}=\int_{0}^{T} \frac{\left(M_{v}^{f}(t) e(t)\right)^{2}}{R_{v}^{\beta}(t)-R_{v}^{\varphi}(t)} d t, \\
K_{x u}^{f}=\int_{0}^{T} \frac{\left(L_{x u}^{f}(t) e(t)\right)^{2}}{R_{u}^{\alpha}(t)-R_{u}^{\varphi}(t)} d t, \quad K_{x v}^{f}=\int_{0}^{T} \frac{\left(L_{x v}^{f}(t) e(t)\right)^{2}}{R_{v}^{\beta}(t)-R_{v}^{\varphi}(t)} d t, \\
K_{x u}^{\varphi}=\int_{0}^{T} \frac{\left(L_{x u}^{\varphi}(t)\right)^{2}}{R_{u}^{\alpha}(t)-R_{u}^{\varphi}(t)} d t, \quad K_{x v}^{\varphi}=\int_{0}^{T} \frac{\left(L_{x v}^{\varphi}(t)\right)^{2}}{R_{v}^{\beta}(t)-R_{v}^{\varphi}(t)} d t, \\
K_{x x}^{f}=\int_{0}^{T} L_{x x}^{f}(t) e(t) d t, \quad K_{x x}^{+\varphi}=\int_{0}^{T} R_{x}^{+\varphi}(t) d t, \quad K_{x x}^{-\varphi}=\int_{0}^{T} R_{x}^{-\varphi}(t) d t ;
\end{gathered}
$$

определим константу $K^{\gamma} \in \mathbb{R} \cup\{+\infty\}$ :

$$
K^{\gamma}=N^{\gamma}+\int_{0}^{T} N_{x}^{\varphi}(t) d t
$$

Будем предполагать, что либо динамика системы (3.1) линейна:

$$
f(t, x, u, v)=A(t) x+B(t) u+C(t) v
$$

либо $K^{\gamma}<+\infty$. 
Определим константы

$$
\begin{aligned}
K_{u u}^{J} & =K^{\gamma}\left(K_{u u}^{f}+2\left(K_{x u}^{f} K_{u}^{f}\right)^{1 / 2}+K_{x x}^{f} K_{u}^{f}\right)+2\left(K_{x u}^{\varphi} K_{u}^{f}\right)^{1 / 2}+K_{x x}^{+\varphi} K_{u}^{f}+R^{+\gamma} K_{u}^{f}, \\
K_{v v}^{J} & =K^{\gamma}\left(K_{v v}^{f}+2\left(K_{x v}^{f} K_{v}^{f}\right)^{1 / 2}+K_{x x}^{f} K_{v}^{f}\right)+2\left(K_{x v}^{\varphi} K_{v}^{f}\right)^{1 / 2}+K_{x x}^{-\varphi} K_{v}^{f}+R^{-\gamma} K_{v}^{f} .
\end{aligned}
$$

Положим по определению, что $(+\infty) \cdot 0=0 \cdot(+\infty)=0$. Поэтому в случае линейной динамики (3.3) первые слагаемые в (3.4) равны 0, даже если $K^{\gamma}=+\infty$.

Наконец, будем предполагать, что

$$
K_{u u}^{J}<1, \quad K_{v v}^{J}<1
$$

\section{4. Известные результаты для дифференциальных игр с сильно выпук-} ло-вогнутым интегрантом. Пусть $\left(t_{0}, x_{0}\right) \in \Omega, u \in \mathscr{U}, v \in \mathscr{V}$. Обозначим решение задачи Коши (3.1) $x(t)$ через $\chi\left(t, t_{0}, x_{0}, u, v\right)$. Определим функционал качества игры:

$$
\begin{aligned}
\mathscr{J}\left(t_{0}, x_{0}, u, v\right)= & \gamma\left(\chi\left(T, t_{0}, x_{0}, u, v\right)\right) \\
& +\int_{t_{0}}^{T}\left(\varphi\left(t, \chi\left(t, t_{0}, x_{0}, u, v\right), u(t), v(t)\right)+\alpha(t, u(t))-\beta(t, v(t))\right) d t .
\end{aligned}
$$

Неравенства (3.5) обеспечивают сильную выпукло-вогнутость функционала $(u, v) \mapsto$ $\mathscr{J}\left(t_{0}, x_{0}, u, v\right)$ для любых $\left(t_{0}, x_{0}\right) \in \Omega$, что вместе с другими предположениями п. 3 позволяет получить следующие две теоремы, доказанные в [5].

ТЕОремА 4.1. Для дифференииальной игры (3.1), (3.2) существует седловая точка в классе программных стратегий: для любых $\left(t_{0}, x_{0}\right) \in \Omega$ найдутся $\widehat{u} \in \mathscr{U}$, $\widehat{v} \in \mathcal{V}$ такие, что для всех $u \in \mathscr{U}, v \in \mathscr{V}$

$$
\mathscr{J}\left(t_{0}, x_{0}, \widehat{u}, v\right) \leqslant \mathscr{J}\left(t_{0}, x_{0}, \widehat{u}, \widehat{v}\right) \leqslant \mathscr{J}\left(t_{0}, x_{0}, u, \widehat{v}\right)
$$

Если $(\widehat{u}, \widehat{v})$ - седловая точка функционала качества игры $(3.1),(3.2)$, то функции $\widehat{u}(\cdot)$ и $\widehat{v}(\cdot)$ будем называть оптимальнымм программньми стратегиями игроков для начальной позиции $\left(t_{0}, x_{0}\right)$.

Из теоремы 4.1 следует, что функция цены игры $\Upsilon$ (см. [3, с. 78]) может быть определена следующей формулой:

$$
\Upsilon\left(t_{0}, x_{0}\right)=\min _{u \in \mathscr{U}} \max _{v \in \mathscr{V}} \mathscr{J}\left(t_{0}, x_{0}, u, v\right)=\max _{v \in \mathscr{V}} \min _{u \in \mathscr{U}} \mathscr{J}\left(t_{0}, x_{0}, u, v\right)
$$

Пусть $t \in[0, T], x \in X(t), u \in P(t), v \in Q(t), \psi \in \mathbb{R}^{1 \times n}$. Определим функцию Понтрягина

$$
\mathscr{H}(t, x, u, v, \psi)=\psi f(t, x, u, v)+\varphi(t, x, u, v)+\alpha(t, u)-\beta(t, v)
$$


Теорема 4.2. Для того чтобы программные стратегии $\widehat{u} \in \mathscr{U}, \widehat{v} \in \mathcal{V}$ были оптимальными, необходимо и достаточно, чтобы существовала абсолютно непрерывная функиия $\psi:\left[t_{0}, T\right] \rightarrow \mathbb{R}^{1 \times n}$ такая, что почти всюду на отрезке $t \in\left[t_{0}, T\right]$ выполнено

$$
\begin{gathered}
\frac{d \psi(t)}{d t}=-\frac{\partial \mathscr{H}}{\partial x}(t, \widehat{x}(t), \widehat{u}(t), \widehat{v}(t), \psi(t)), \quad \psi(T)=\frac{d \gamma}{d x}(\widehat{x}(T)), \\
\frac{d \widehat{x}(t)}{d t}=\frac{\partial \mathscr{H}}{\partial \psi}(t, \widehat{x}(t), \widehat{u}(t), \widehat{v}(t), \psi(t)), \quad \widehat{x}\left(t_{0}\right)=x_{0}, \\
\mathscr{H}(t, \widehat{x}(t), \widehat{u}(t), v, \psi(t)) \leqslant \mathscr{H}(t, \widehat{x}(t), \widehat{u}(t), \widehat{v}(t), \psi(t)) \leqslant \mathscr{H}(t, \widehat{x}(t), u, \widehat{v}(t), \psi(t))
\end{gathered}
$$

для всех $u \in P(t), v \in Q(t)$.

\section{5. Непрерывность оптимальных стратегий и гладкость функции цены иг- ры.}

ОПРЕДЕЛЕНИЕ 5.1. Пусть $\widehat{u}, \widehat{v}$ - оптимальные программные стратегии игроков для начальной позиции $\left(t_{0}, x_{0}\right) \in \Omega$. Функцию $\widehat{x}:\left[t_{0}, T\right] \rightarrow \mathbb{R}^{n}$, являющуюся решением задачи Коши (4.4), будем называть оптимальной траекторией фазового вектора для начальной позиции $\left(t_{0}, x_{0}\right)$, а функцию $\psi:\left[t_{0}, T\right] \rightarrow \mathbb{R}^{1 \times n}$, являющуюся решением задачи Коши (4.3), - оптимальной траекторией сопряженного вектора для начальной позиции $\left(t_{0}, x_{0}\right)$.

ТЕОремА 5.1. Для любой начальной позииии $\left(t_{0}, x_{0}\right) \in \Omega$ оптимальные программные стратегии $\widehat{u}(\cdot), \widehat{v}(\cdot)$ являются непрерывными функциями на отрезке $\left[t_{0}, T\right]$.

ДокАЗАТЕЛЬСтво. Пусть $\widehat{u}(\cdot), \widehat{v}(\cdot)$ - оптимальные программные стратегии игроков, $\widehat{x}(\cdot), \psi(\cdot)$ - оптимальные траектории фазового и сопряженного векторов для начальной позиции $\left(t_{0}, x_{0}\right) \in \Omega$. Из (4.3) следует, что для почти всех $t \in\left[t_{0}, T\right]$

$$
\left\|\frac{d \psi(t)}{d t}\right\| \leqslant\|\psi(t)\| M_{x}^{f}(t)+N_{x}^{\varphi}(t), \quad\|\psi(T)\| \leqslant N^{\gamma} .
$$

Поэтому для всех $t \in\left[t_{0}, T\right]$

$$
\|\psi(t)\| \leqslant K^{\gamma} e(t) .
$$

Для любых $t \in\left[t_{0}, T\right], u \in P_{0}, v \in Q_{0}$ обозначим $\mu(t, u, v)=\psi(t) f(t, \widehat{x}(t), u, v)+$ $\varphi(t, \widehat{x}(t), u, v)$. Из предположений п. 3 и неравенства (5.1) следует вьполнение условий (2.3) теоремы 2.2 для любых $\omega=t \in \Omega=\left[t_{0}, T\right], u \in P_{0}, v \in Q_{0}$, где

$$
R_{u}^{\mu}(t)=L_{u u}^{f}(t) K^{\gamma} e(t)+R_{u}^{\varphi}(t), \quad R_{v}^{\mu}(t)=L_{v v}^{f}(t) K^{\gamma} e(t)+R_{v}^{\varphi}(t)
$$

Отсюда и из определения константы $K_{u u}^{f}$ следует, что для любого $t \in\left[t_{0}, T\right]$ справедливо неравенство

$$
R_{u}^{\alpha}(t)-R_{u}^{\mu}(t) \geqslant\left(R_{u}^{\alpha}(t)-R_{u}^{\varphi}(t)\right)\left(1-K^{\gamma} K_{u u}^{f}\right)
$$


что вместе с условиями (3.4) и (3.5) дает неравенство

$$
R_{u}^{\alpha}(t)-R_{u}^{\mu}(t) \geqslant R_{u}^{\min }\left(1-K_{u u}^{J}\right)>0 .
$$

Аналогично, для любого $t \in\left[t_{0}, T\right]$ справедливо неравенство

$$
R_{v}^{\beta}(t)-R_{v}^{\mu}(t) \geqslant R_{v}^{\min }\left(1-K_{v v}^{J}\right)>0 .
$$

Тем самым, выполнены условия (2.8) теоремы 2.3. Из предположений п. 3 легко получить вьполнение остальных условий утверждения а) теоремы 2.3, применение которого дает требуемое утверждение.

ТЕОрема 5.2. Для любого $t_{0} \in[0, T]$ функиия иены игры $\Upsilon\left(t_{0}, \cdot\right)$ липшичево дифферениируема на множестве $X\left(t_{0}\right)$ с константой, не зависящей от $t_{0}, u$ для любого $x_{0} \in X\left(t_{0}\right)$ справедливо равенство

$$
\frac{\partial \Upsilon}{\partial x}\left(t_{0}, x_{0}\right)=\psi\left(t_{0}\right)
$$

где $\psi(\cdot)$ - траектория сопряжснного вектора для начальной позичии $\left(t_{0}, x_{0}\right)$.

ДокАЗАТЕЛЬСТвО. Пусть $\widehat{u}(\cdot), \widehat{v}(\cdot)$ - оптимальные программные стратегии игроков, а $\widehat{x}(\cdot)$ - оптимальная траектория фазового вектора для начальной позиции $\left(t_{0}, x_{0}\right)$. Для любого вектора $\eta \in X\left(t_{0}\right)$ обозначим через $u^{\eta}(\cdot), v^{\eta}(\cdot)$ оптимальные программные стратегии игроков, а через $x^{\eta}(\cdot)$ оптимальную траекторию фазового вектора для начальной позиции $\left(t_{0}, \eta\right)$. Тогда $\Upsilon\left(t_{0}, \eta\right)=\mathscr{J}\left(t_{0}, \eta, u^{\eta}, v^{\eta}\right), \Upsilon\left(t_{0}, x_{0}\right)=\mathscr{J}\left(t_{0}, x_{0}, \widehat{u}, \widehat{v}\right)$. Поскольку $\mathscr{J}\left(t_{0}, \eta, u^{\eta}, v^{\eta}\right) \geqslant \mathscr{J}\left(t_{0}, \eta, u^{\eta}, \widehat{v}\right)$, вьполнено

$$
\Upsilon\left(t_{0}, \eta\right)-\Upsilon\left(t_{0}, x_{0}\right) \geqslant \mathscr{J}\left(t_{0}, \eta, u^{\eta}, \widehat{v}\right)-\mathscr{J}\left(t_{0}, \eta, \widehat{u}, \widehat{v}\right)
$$

Введем обозначения

$$
\begin{gathered}
\varphi_{x}(t)=\frac{\partial \varphi}{\partial x}(t, \widehat{x}(t), \widehat{u}(t), \widehat{v}(t)), \quad f_{x}(t)=\frac{\partial f}{\partial x}(t, \widehat{x}(t), \widehat{u}(t), \widehat{v}(t)), \\
f_{u}(t)=\frac{\partial f}{\partial u}(t, \widehat{x}(t), \widehat{u}(t), \widehat{v}(t))
\end{gathered}
$$

Пусть для любого $\tau \in\left[t_{0}, T\right]$ функция $t \mapsto \Phi(t, \tau) \in \mathbb{R}^{n \times n}$ является решением задачи Коши

$$
\frac{\partial \Phi(t, \tau)}{\partial t}=-\Phi(t, \tau) f_{x}(t), \quad t \in\left[t_{0}, T\right], \quad \Phi(\tau, \tau)=E
$$

Из уравнения (4.3) следует, что для любого $t \in\left[t_{0}, T\right]$ справедлива формула

$$
\psi(t)=\frac{d \gamma}{d x}(\widehat{x}(T)) \Phi(t, T)+\int_{t}^{T} \varphi_{x}(\tau) \Phi(t, \tau) d \tau
$$


Для любой допустимой программной стратегии $u(\cdot) \in \mathscr{U}$ определим

$$
\rho(u)=\left(\int_{t_{0}}^{T}\left(R_{u}^{\alpha}(t)-R_{u}^{\varphi}(t)\right)\|u(t)\|^{2} d t\right)^{1 / 2}
$$

В силу леммы 2 из [5] и формулы (5.4) существуют константы $K_{u \eta}^{J}$ и $K_{\eta \eta}^{+J}$ такие, что для любой допустимой программной стратегии $u(\cdot) \in \mathscr{U}$ справедливо неравенство

$$
\begin{aligned}
& \mathscr{J}\left(t_{0}, \eta, u, \widehat{v}\right)-\mathscr{J}\left(t_{0}, \eta, \widehat{u}, \widehat{v}\right) \\
& \geqslant \int_{t_{0}}^{T}(\varphi(t, \widehat{x}(t), u(t), \widehat{v}(t))+\alpha(t, u(t))-\varphi(t, \widehat{x}(t), \widehat{u}(t), \widehat{v}(t))-\alpha(t, \widehat{u}(t)) \\
& \left.\quad+\psi(t) f_{u}(t)(u(t)-\widehat{u}(t))\right) d t \\
& \quad+\psi\left(t_{0}\right)\left(\eta-x_{0}\right)-\frac{1}{2} K_{u u}^{J} \rho^{2}(u-\widehat{u})-K_{u \eta}^{J} \rho(u-\widehat{u})\left\|\eta-x_{0}\right\|-\frac{1}{2} K_{\eta \eta}^{+J}\left\|\eta-x_{0}\right\|^{2} .
\end{aligned}
$$

Из правой части неравенства (4.5) следует, что $0 \in \partial_{u} \mathscr{H}(t, \widehat{x}(t), \widehat{u}(t), \widehat{v}(t), \psi(t))$ для любого $t \in\left[t_{0}, T\right]$. Поэтому

$$
\psi(t) f_{u}(t) \in-\partial_{u}(\varphi(t, \widehat{x}(t), \widehat{u}(t), \widehat{v}(t))+\alpha(t, \widehat{u}(t))) .
$$

Из этого условия и условий $\varphi(t, x, \cdot, v) \in \mathrm{WCo}\left(P(t) ; R_{u}^{\varphi}(t)\right), \alpha(t, \cdot) \in \mathrm{SCo}\left(P(t) ; R_{u}^{\alpha}(t)\right)$ получаем неравенство

$$
\begin{aligned}
\int_{t_{0}}^{T}(\varphi(t, \widehat{x}(t), u(t), \widehat{v}(t))+\alpha(t, u(t))-\varphi(t, \widehat{x}(t), \widehat{u}(t), \widehat{v}(t)) \\
\left.\quad-\alpha(t, \widehat{u}(t))+\psi(t) f_{u}(t)(u(t)-\widehat{u}(t))\right) d t \\
\geqslant \frac{1}{2} \int_{t_{0}}^{T}\left(R_{u}^{\alpha}(t)-R_{u}^{\varphi}(t)\right)\|u(t)-\widehat{u}(t)\|^{2} d t=\frac{1}{2} \rho^{2}(u-\widehat{u}) .
\end{aligned}
$$

Из неравенств (5.3), (5.5), (5.6) следует неравенство

$$
\begin{aligned}
\Upsilon\left(t_{0}, \eta\right)-\Upsilon\left(t_{0}, x_{0}\right) \geqslant & \psi\left(t_{0}\right)\left(\eta-x_{0}\right)+\frac{1}{2}\left(1-K_{u u}^{J}\right) \rho^{2}\left(u^{\eta}-\widehat{u}\right) \\
& -K_{u \eta}^{J} \rho\left(u^{\eta}-\widehat{u}\right)\left\|\eta-x_{0}\right\|-\frac{1}{2} K_{\eta \eta}^{+J}\left\|\eta-x_{0}\right\|^{2}
\end{aligned}
$$

Обозначая

$$
R_{x}^{+\Upsilon}=K_{\eta \eta}^{+J}+\frac{\left(K_{u \eta}^{J}\right)^{2}}{1-K_{u u}^{J}}
$$


получим

$$
\begin{aligned}
\Upsilon\left(t_{0}, \eta\right)-\Upsilon\left(t_{0}, x_{0}\right) \geqslant & \psi\left(t_{0}\right)\left(\eta-x_{0}\right)+\frac{1}{2}\left(1-K_{u u}^{J}\right)\left(\rho\left(u^{\eta}-\widehat{u}\right)-\left\|\eta-x_{0}\right\| \frac{K_{u \eta}^{J}}{1-K_{u u}^{J}}\right)^{2} \\
& -\frac{1}{2} R_{x}^{+\Upsilon}\left\|\eta-x_{0}\right\|^{2}
\end{aligned}
$$

Следовательно,

$$
\Upsilon\left(t_{0}, \eta\right)-\Upsilon\left(t_{0}, x_{0}\right) \geqslant \psi\left(t_{0}\right)\left(\eta-x_{0}\right)-\frac{1}{2} R_{x}^{+\Upsilon}\left\|\eta-x_{0}\right\|^{2}
$$

Аналогично, существует константа $R_{x}^{-\Upsilon}$ такая, что для любого $\eta \in X\left(t_{0}\right)$ справедливо неравенство

$$
\Upsilon\left(t_{0}, \eta\right)-\Upsilon\left(t_{0}, x_{0}\right) \leqslant \psi\left(t_{0}\right)\left(\eta-x_{0}\right)+\frac{1}{2} R_{x}^{-\Upsilon}\left\|\eta-x_{0}\right\|^{2}
$$

Из (5.7), (5.8) следует неравенство

$$
\left|\Upsilon\left(t_{0}, \eta\right)-\Upsilon\left(t_{0}, x_{0}\right)-\psi\left(t_{0}\right)\left(\eta-x_{0}\right)\right| \leqslant \frac{1}{2} R_{x}^{\Upsilon}\left\|\eta-x_{0}\right\|^{2}
$$

где $R_{x}^{\Upsilon}=\max \left\{R_{x}^{+\Upsilon}, R_{x}^{-\Upsilon}\right\}$.

Из последнего неравенства следует дифференцируемость функции $\Upsilon\left(t_{0}, \cdot\right)$ в точке $x_{0}$ и равенство (5.2). Из неравенств (5.7), (5.8) соответственно легко получить условия $\Upsilon\left(t_{0}, \cdot\right) \in \mathrm{WCo}\left(X\left(t_{0}\right) ; R_{x}^{+\Upsilon}\right), \Upsilon\left(t_{0}, \cdot\right) \in-\mathrm{WCo}\left(X\left(t_{0}\right) ; R_{x}^{-\Upsilon}\right)$, из которых в силу теоремы 1.1 следует липшицева дифференцируемость функции $\Upsilon\left(t_{0}, \cdot\right)$ на множестве $X\left(t_{0}\right)$ с константой $R_{x}^{\Upsilon}$.

Заметим, что липшищева дифференцируемость функции $\Upsilon\left(t_{0}, \cdot\right)$ также следует непосредственно из теоремы 2 из [5] и теоремы 1.1.

Лемма 5.1. Для любьи $t_{0} \in(0, T], x_{0} \in X\left(t_{0}\right)$ существуют число $t_{*} \in\left[0, t_{0}\right) u$ вектор $x_{*} \in X\left(t_{*}\right)$ такие, что оптимальная траектория $\widehat{x}(\cdot)$ фазового вектора для начальной позичии $\left(t_{*}, x_{*}\right)$ удовлетворяет условию $\widehat{x}\left(t_{0}\right)=x_{0}$.

ДокАЗАТЕЛЬСтво. Определим множество

$$
W=\left\{(t, x, \psi): t \in[0, T], x \in X(t), \psi \in \mathbb{R}^{1 \times n},\|\psi\| \leqslant K^{\gamma} e(t)\right\}
$$

Следуя рассуждениям при доказательстве теоремы 5.1, легко видеть, что для любых $(t, x, \psi) \in W$ функция Понтрягина $(u, v) \mapsto \mathscr{H}(t, x, u, v, \psi)$ сильно вьпукло-вогнута и имеет седловую точку, которую обозначим через $\left(u^{H}(t, x, \psi), v^{H}(t, x, \psi)\right)$. Из утверждения а) теоремы 2.3 легко получить, что функции $(t, x, \psi) \mapsto u^{H}(t, x, \psi),(t, x, \psi) \mapsto$ $v^{H}(t, x, \psi)$ непрерьвны на множестве $W$. 
Для любых $(t, x, \psi) \in W$ определим

$$
\begin{gathered}
F(t, x, \psi)=f\left(t, x, u^{H}(t, x, \psi), v^{H}(t, x, \psi)\right), \\
F_{1}(t, x, \psi)=\frac{\partial f}{\partial x}\left(t, x, u^{H}(t, x, \psi), v^{H}(t, x, \psi)\right) .
\end{gathered}
$$

Пусть $\widehat{x}:\left[t_{0}, T\right] \rightarrow \mathbb{R}^{n}$ и $\psi:\left[t_{0}, T\right] \rightarrow \mathbb{R}^{1 \times n}$ - оптимальные траектории фазового и сопряженного векторов для начальной позиции $\left(t_{0}, x_{0}\right)$. Обозначим $\psi_{0}=\psi\left(t_{0}\right)$ и рассмотрим задачу Коши

$$
\begin{aligned}
\frac{d \widehat{x}(t)}{d t} & =F(t, \widehat{x}(t), \psi(t)), \quad \widehat{x}\left(t_{0}\right)=x_{0}, \\
\frac{d \psi(t)}{d t} & =-\psi(t) F_{1}(t, \widehat{x}(t), \psi(t)), \quad \psi\left(t_{0}\right)=\psi_{0} .
\end{aligned}
$$

Из непрерьвности функций $u^{H}(\cdot), v^{H}(\cdot)$ и предположений п. 3 следует непрерывность функций $F(\cdot)$ и $F_{1}(\cdot)$ на множестве $W$. Поэтому в некоторой левой полуокрестности точки $t_{0}$ существуют функции $\widehat{x}(\cdot), \psi(\cdot)$, являюшиеся решением задачи Коши (5.9). Выберем точку $t_{*}<t_{0}$ из этой полуокрестности. Из теоремы 4.2 следует, что функция $\widehat{x}:\left[t_{*}, T\right] \rightarrow \mathbb{R}^{n}$ является оптимальной траекторией фазового вектора для начальной позиции $\left(t_{*}, x_{*}\right)$, где $x_{*}=\widehat{x}\left(t_{*}\right)$.

ЛЕмма 5.2. Если $\widehat{u}(\cdot), \widehat{v}(\cdot)$ - оптимальные программнье стратегии игроков, a $\widehat{x}(\cdot)$ - оптимальная траектория фазового вектора для некоторой начальной позичии $\left(t_{0}, x_{0}\right) \in \Omega$, то функция

$$
t \mapsto \varphi(t, \widehat{x}(t), \widehat{u}(t), \widehat{v}(t))+\alpha(t, \widehat{u}(t))-\beta(t, \widehat{v}(t))
$$

непрерывна на $\left[t_{0}, T\right]$.

ДокАЗАТЕльСтво. Пусть $\psi(\cdot)$ - оптимальная траектория сопряженного вектора для начальной позиции $\left(t_{0}, x_{0}\right)$. Из правой части неравенства (4.5) теоремы 4.2 следует, что для любого $t \in\left[t_{0}, T\right]$ справедливо включение $0 \in \partial_{u} \mathscr{H}(t, \widehat{x}(t), \widehat{u}(t), \widehat{v}(t), \psi(t))$, т.е.

$$
\psi(t) \frac{\partial f}{\partial u}(t, \widehat{x}(t), \widehat{u}(t), \widehat{v}(t))+\frac{\partial \varphi}{\partial u}(t, \widehat{x}(t), \widehat{u}(t), \widehat{v}(t)) \in-\partial_{u} \alpha(t, \widehat{u}(t))
$$

Для любых $t \in\left[t_{0}, T\right], \xi^{u} \in \mathbb{R}^{1 \times p}$ определим

$$
\begin{gathered}
\widehat{\xi}_{u}(t)=-\psi(t) \frac{\partial f}{\partial u}(t, \widehat{x}(t), \widehat{u}(t), \widehat{v}(t))-\frac{\partial \varphi}{\partial u}(t, \widehat{x}(t), \widehat{u}(t), \widehat{v}(t)), \\
\alpha^{*}\left(t, \xi^{u}\right)=\max _{u \in P(t)}\left(\xi^{u} u-\alpha(t, u)\right) .
\end{gathered}
$$


Из условия $\widehat{\xi}_{u}(t) \in \partial_{u} \alpha(t, \widehat{u}(t))$ следует, что максимум достигается на векторе $u=\widehat{u}(t)$. В силу предположений п. 3 и теоремы 5.1 функция $\widehat{\xi}_{u}(\cdot)$ непрерывна на $\left[t_{0}, T\right]$.

Заметим, что для любого $t \in\left[t_{0}, T\right]$ функция $\alpha^{*}(t, \cdot)$ удовлетворяет условию Липшища на множестве $\mathbb{R}^{1 \times p}$ с константой $\max _{t \in\left[t_{0}, T\right]}\|\widehat{u}(t)\|$. Согласно предположению п. 3 для любого $\xi^{u} \in \mathbb{R}^{1 \times p}$ функция $\alpha^{*}\left(\cdot, \xi^{u}\right)$ непрерывна. Поэтому функция $t \mapsto \alpha^{*}\left(t, \widehat{\xi}_{u}(t)\right)$ непрерьвна, откуда следует непрерьвность функции $t \mapsto \alpha(t, \widehat{u}(t))=\widehat{\xi}_{u}(t) \widehat{u}(t)-$ $\alpha^{*}\left(t, \widehat{\xi}_{u}(t)\right)$. Аналогично, функция $t \mapsto \beta(t, \widehat{v}(t))$ непрерьвна на отрезке $\left[t_{0}, T\right]$. Непрерьвность функции $t \mapsto \varphi(t, \widehat{x}(t), \widehat{u}(t), \widehat{v}(t))$ следует из теоремы 5.1 и непрерывности функций $\widehat{x}(\cdot)$ и $\varphi(\cdot)$.

ЛЕмма 5.3. Функиия $\partial \Upsilon / \partial x$ непрерывна на множестве $\Omega$.

ДокАЗАТЕЛЬСТво. Зафиксируем произвольную начальную позищию $\left(t_{0}, x_{0}\right) \in \Omega$. Если $t_{0}>0$, то в силу леммы 5.1 существуют число $t_{*} \in\left[0, t_{0}\right)$ и вектор $x_{*} \in X\left(t_{*}\right)$ такие, что оптимальная траектория фазового вектора $\widehat{x}(\cdot)$ для начальной позиции $\left(t_{*}, x_{*}\right)$ удовлетворяет условию $\widehat{x}\left(t_{0}\right)=x_{0}$. Если $t_{0}=0$, то положим $\left(t_{*}, x_{*}\right)=\left(t_{0}, x_{0}\right)$.

В силу теоремы 5.2 функция $x \mapsto \partial \Upsilon(t, x) / \partial x$ удовлетворяет условию Липшица на множестве $X(t)$ с константой, не зависящей от $t$. Поэтому

$$
\left\|\frac{\partial \Upsilon}{\partial x}(t, \widehat{x}(t))-\frac{\partial \Upsilon}{\partial x}(t, x)\right\| \rightarrow 0 \quad \text { при } \quad(t, x) \rightarrow\left(t_{0}, x_{0}\right) .
$$

Из теоремы 5.2 также следует, что

$$
\left\|\frac{\partial \Upsilon}{\partial x}(t, \widehat{x}(t))-\frac{\partial \Upsilon}{\partial x}\left(t_{0}, x_{0}\right)\right\|=\left\|\psi(t)-\psi\left(t_{0}\right)\right\| \rightarrow 0 \quad \text { при } t \rightarrow t_{0},
$$

где $\psi(\cdot)$ - оптимальная траектория сопряженного вектора для начальной позиции $\left(t_{*}, x_{*}\right)$.

Поэтому

$$
\left\|\frac{\partial \Upsilon}{\partial x}(t, x)-\frac{\partial \Upsilon}{\partial x}\left(t_{0}, x_{0}\right)\right\| \rightarrow 0 \quad \text { при }(t, x) \rightarrow\left(t_{0}, x_{0}\right) .
$$

ЛЕмма 5.4. Пусть задан отрезок $\Delta \subset \mathbb{R}$ и функиия $g: \Delta \times \Delta \rightarrow \mathbb{R}$ такая, что для любого $t \in \Delta$ функиии $\tau \mapsto g(t, \tau), t \mapsto g(t, t)$ дифференцируемы на отрезке $\Delta$ и функиия $(t, \tau) \mapsto \partial g(t, \tau) / \partial \tau$ непрерывна на множестве $\Delta \times \Delta$. Тогда для любого $t_{0} \in \Delta$ функиия $t \mapsto g\left(t, t_{0}\right)$ дифференцируема в точке $t=t_{0}$ и справедливо равенство

$$
\left.\frac{\partial g(t, \tau)}{\partial t}\right|_{(t, \tau)=\left(t_{0}, t_{0}\right)}=\left.\frac{d}{d t} g(t, t)\right|_{t=t_{0}}-\left.\frac{\partial g(t, \tau)}{\partial \tau}\right|_{(t, \tau)=\left(t_{0}, t_{0}\right)} .
$$


ДокАЗАТЕЛЬСТво. По теореме о среднем для любого числа $\delta$, удовлетворяющего включению $t_{0}+\delta \in \Delta$, существует число $\theta \in[0,1]$ такое, что

$$
\frac{g\left(t_{0}+\delta, t_{0}\right)-g\left(t_{0}+\delta, t_{0}+\delta\right)}{\delta}=-\left.\frac{\partial g(t, \tau)}{\partial \tau}\right|_{(t, \tau)=\left(t_{0}+\delta, t_{0}+\theta \delta\right)} \stackrel{\delta \rightarrow 0}{\rightarrow}-\left.\frac{\partial g(t, \tau)}{\partial \tau}\right|_{(t, \tau)=\left(t_{0}, t_{0}\right)}
$$

Кроме того,

$$
\left.\frac{g\left(t_{0}+\delta, t_{0}+\delta\right)-g\left(t_{0}, t_{0}\right)}{\delta} \stackrel{\delta \rightarrow 0}{\rightarrow} \frac{d g(t, t)}{d t}\right|_{t=t_{0}}
$$

Следовательно,

$$
\left.\frac{g\left(t_{0}+\delta, t_{0}\right)-g\left(t_{0}, t_{0}\right)}{\delta} \stackrel{\delta \rightarrow 0}{\rightarrow} \frac{d g(t, t)}{d t}\right|_{t=t_{0}}-\left.\frac{\partial g(t, \tau)}{\partial \tau}\right|_{(t, \tau)=\left(t_{0}, t_{0}\right)}
$$

ТЕОрема 5.3. Функиия иены игры непрерывно дифферениируема на множестве $\Omega$ и для любих $(t, x) \in \Omega$ справедливо уравнение Айзекса-Беллмана (уравнение дифференииального минимакса [3, с. 138]):

$$
-\frac{\partial \Upsilon(t, x)}{\partial t}=\min _{u \in P(t)} \max _{v \in Q(t)}\left(\frac{\partial \Upsilon(t, x)}{\partial x} f(t, x, u, v)+\varphi(t, x, u, v)+\alpha(t, u)-\beta(t, v)\right) .
$$

ДокАЗАТЕЛьСтво. Зафиксируем произвольную начальную позицию $\left(t_{0}, x_{0}\right) \in \Omega$. Если $t_{0}>0$, то в силу леммы 5.1 существуют число $t_{*} \in\left[0, t_{0}\right)$ и вектор $x_{*} \in X\left(t_{*}\right)$ такие, что оптимальная траектория фазового вектора $\widehat{x}(\cdot)$ для начальной позиции $\left(t_{*}, x_{*}\right)$ удовлетворяет условию $\widehat{x}\left(t_{0}\right)=x_{0}$. Если $t_{0}=0$, то положим $\left(t_{*}, x_{*}\right)=\left(t_{0}, x_{0}\right)$. Пусть $\widehat{u}(\cdot), \widehat{v}(\cdot)$ - оптимальные программные стратегии игроков, а $\psi(\cdot)$ - оптимальная траектория сопряженного вектора для начальной позиции $\left(t_{*}, x_{*}\right)$. По определению функции цены игры для любого $t \in\left[t_{*}, T\right]$ справедливо равенство

$$
\Upsilon(t, \widehat{x}(t))=\gamma(\widehat{x}(T))+\int_{t}^{T}(\varphi(\tau, \widehat{x}(\tau), \widehat{u}(\tau), \widehat{v}(\tau))+\alpha(\tau, \widehat{u}(\tau))-\beta(\tau, \widehat{v}(\tau))) d \tau
$$

Отсюда и из леммы 5.2 следует, что функция $t \mapsto \Upsilon(t, \widehat{x}(t))$ дифференцируема на отрезке $\left[t_{*}, T\right]$ и справедливо равенство

$$
-\frac{d}{d t} \Upsilon(t, \widehat{x}(t))=\varphi(t, \widehat{x}(t), \widehat{u}(t), \widehat{v}(t))+\alpha(t, \widehat{u}(t))-\beta(t, \widehat{v}(t)) .
$$

Поскольку множество $\Omega$ открыто, а функция $\widehat{x}(\cdot)$ непрерьвна, то существует отрезок $\Delta \subset\left[t_{*}, T\right], t_{0} \in \operatorname{int}(\Delta)$ такой, что для любых $(t, \tau) \in \Delta \times \Delta$ имеет место включение $(t, \widehat{x}(\tau)) \in \Omega$. При $(t, \tau) \in \Delta \times \Delta$ определим $g(t, \tau)=\Upsilon(t, \widehat{x}(\tau))$.

В силу леммы 5.3 функция

$$
(t, \tau) \mapsto \frac{\partial g(t, \tau)}{\partial \tau}=\frac{\partial \Upsilon}{\partial x}(t, \widehat{x}(\tau)) f(\tau, \widehat{x}(\tau), \widehat{u}(\tau), \widehat{v}(\tau))
$$


непрерьвна на множестве $\Delta \times \Delta$.

Применяя лемму 5.4 и формулу (5.10), получаем равенство

$$
\begin{aligned}
-\frac{\partial \Upsilon}{\partial t}\left(t_{0}, x_{0}\right)= & \frac{\partial \Upsilon}{\partial x}\left(t_{0}, x_{0}\right) f\left(t_{0}, x_{0}, \widehat{u}\left(t_{0}\right), \widehat{v}\left(t_{0}\right)\right) \\
& +\varphi\left(t_{0}, x_{0}, \widehat{u}\left(t_{0}\right), \widehat{v}\left(t_{0}\right)\right)+\alpha\left(t_{0}, \widehat{u}\left(t_{0}\right)\right)-\beta\left(t_{0}, \widehat{v}\left(t_{0}\right)\right) .
\end{aligned}
$$

Поскольку согласно теореме 5.2

$$
\frac{\partial \Upsilon}{\partial x}\left(t_{0}, x_{0}\right)=\psi\left(t_{0}\right)
$$

то в силу теоремы 4.2 формулу (5.11) можно переписать в виде

$$
\begin{aligned}
-\frac{\partial \Upsilon}{\partial t}\left(t_{0}, x_{0}\right)= & \min _{u \in P\left(t_{0}\right)} \max _{v \in Q\left(t_{0}\right)}\left(\frac{\partial \Upsilon}{\partial x}\left(t_{0}, x_{0}\right) f\left(t_{0}, x_{0}, u, v\right)\right. \\
& \left.+\varphi\left(t_{0}, x_{0}, u, v\right)+\alpha\left(t_{0}, u\right)-\beta\left(t_{0}, v\right)\right) .
\end{aligned}
$$

Из непрерывности правой части последнего уравнения следует непрерывность функции $\partial \Upsilon / \partial t$ на множестве $\Omega$.

Для любых $(t, x) \in \Omega$ через $\left(u_{\text {pos }}(t, x), v_{\text {pos }}(t, x)\right)$ обозначим седловую точку функции

$$
(u, v) \mapsto \frac{\partial \Upsilon(t, x)}{\partial x} f(t, x, u, v)+\varphi(t, x, u, v)+\alpha(t, u)-\beta(t, v)
$$

Функции $u_{\mathrm{pos}}: \Omega \rightarrow \mathbb{R}^{p}, v_{\mathrm{pos}}: \Omega \rightarrow \mathbb{R}^{q}$ являются оптимальными позиционными стратегиями игроков [3].

ТЕОРема 5.4. Оптимальные позиционные стратегии игроков $u_{\text {pos }}, v_{\text {pos непре- }}$ рывны на множсестве $\Omega$.

ДокАЗАТЕЛЬСТво. Определим функцию $\mu: \Omega \times P_{0} \times Q_{0} \rightarrow \mathbb{R}$ :

$$
\mu(t, x, u, v)=\frac{\partial \Upsilon(t, x)}{\partial x} f(t, x, u, v)+\varphi(t, x, u, v) .
$$

Из теоремы 5.2 и формулы (5.1) получаем неравенство

$$
\left\|\frac{\partial \Upsilon(t, x)}{\partial x}\right\| \leqslant K^{\gamma} e(t), \quad(t, x) \in \Omega .
$$

Отсюда и из предположений п. 3 следуют условия $(2.3),(2.4)$ теоремы 2.2, где $\omega=(t, x)$,

$$
\begin{gathered}
R_{u}^{\mu}(t)=L_{u u}^{f}(t) e(t) K^{\gamma}+R_{u}^{\varphi}(t), \quad R_{v}^{\mu}(t)=L_{v v}^{f}(t) e(t) K^{\gamma}+R_{v}^{\varphi}(t), \\
L_{u u}^{\mu}(t)=L_{u u}^{f}(t) e(t) K^{\gamma}+L_{u u}^{\varphi}(t), \quad L_{u v}^{\mu}(t)=L_{u v}^{f}(t) e(t) K^{\gamma}+L_{u v}^{\varphi}(t), \\
L_{v v}^{\mu}(t)=L_{v v}^{f}(t) e(t) K^{\gamma}+L_{v v}^{\varphi}(t) .
\end{gathered}
$$


Из (3.4) и (3.5) получаем неравенство $K^{\gamma} K_{u u}^{f}<1$, из которого следуют условия (2.8) теоремы 2.3 .

Используя лемму 5.3, получим, что для любых $u \in P_{0}, v \in Q_{0}$ функции

$$
(t, x) \mapsto \frac{\partial \mu}{\partial u}(t, x, u, v), \quad(t, x) \mapsto \frac{\partial \mu}{\partial v}(t, x, u, v)
$$

непрерьвны на множестве $\Omega$. Отсюда и из предположений п. 3 следует, что выполнены все условия утверждения а) теоремы 2.3, применение которого завершает доказательство теоремы.

\section{СПИСОК ЦИТИРОВАННОЙ ЛИТЕРАТУРЫ}

[1] Айзекс Р. Дифференциальные игры. М.: Мир, 1967.

[2] Понтрягин Л.С. Линейные дифференциальные игры преследования // Матем. сб. 1980. Т. 112. № 3. С. 307-330.

[3] Красовский Н. Н. Управление динамической системой. М.: Наука, 1985.

[4] Субботин А.И. Минимаксные неравенства и уравнение Гамильтона-Якоби. М.: Наука, 1991.

[5] Иванов Г. Е. Седловая точка для дифференциальных игр с сильно выпукло-вогнутым интегрантом // Матем. заметки. 1997. Т. 62. №5. С. 725-743.

Московский физико-технический институт 